\title{
Kinematics of the old stellar population at the Galactic centre ${ }^{\star}$
} \author{
T. Ott ${ }^{1}$, K. Dodds-Eden ${ }^{1}$, and R. Genzel ${ }^{1,3}$ \\ 1 Max-Planck-Institut für extraterrestrische Physik, Giessenbachstrasse 1, 85748 Garching, Germany \\ e-mail: trippe@mpe.mpg.de \\ 2 GRAAL-CNRS, Université Montpellier II, Place Eugène Bataillon, 34090 Montpellier, France \\ 3 University of California, 94720 Berkeley, California, USA
}

S. Trippe ${ }^{1}$, S. Gillessen ${ }^{1}$, O. E. Gerhard ${ }^{1}$, H. Bartko ${ }^{1}$, T. K. Fritz ${ }^{1}$, H. L. Maness ${ }^{3}$, F. Eisenhauer ${ }^{1}$, F. Martins ${ }^{2}$,

Received 13 May 2008 / Accepted 6 October 2008

ABSTRACT

\begin{abstract}
Aims. We aim at a detailed description of the kinematic properties of the old, (several Gyrs) late-type CO-absorption star population among the Galactic centre (GC) cluster stars. This cluster is composed of a central supermassive black hole (Sgr A*) and a selfgravitating system of stars. Understanding its kinematics thus offers the opportunity to understand the dynamical interaction between a central point mass and the surrounding stars in general, especially in view of understanding other galactic nuclei.

Methods. We applied AO-assisted, near-infrared imaging and integral-field spectroscopy using the instruments NAOS/CONICA and SINFONI at the VLT. We obtained proper motions for 5445 stars, 3D velocities for 664 stars, and acceleration limits (in the sky plane) for 750 stars. Global kinematic properties were analysed using velocity and velocity dispersion distributions, phase-space maps, twopoint correlation functions, and the Jeans equation.

Results. We detect for the first time significant cluster rotation in the sense of the general Galactic rotation in proper motions. Out of the $3 \mathrm{D}$ velocity dispersion, we derive an improved statistical parallax for the $\mathrm{GC}$ of $R_{0}=8.07 \pm 0.32_{\text {stat }} \pm 0.13_{\text {sys }} \mathrm{kpc}$. The distribution of 3D stellar speeds can be approximated by local Maxwellian distributions. Kinematic modelling provides deprojected 3D kinematic parameters, including the mass profile of the cluster. We find an upper limit of $4 \%$ for the amplitude of fluctuations in the phase-space distribution of the cluster stars compared to a uniform, spherical model cluster. Using upper limits on accelerations, we constrain the minimum line-of-sight distances from the plane of Sgr A* of five stars located within the innermost few (projected) arcsec. The stars within $0.7^{\prime \prime}$ radius from the star group IRS13E do not co-move with this group, making it unlikely that IRS13E is the core of a substantial star cluster. Overall, the GC late-type cluster is described well as a uniform, isotropic, rotating, dynamically relaxed, phase-mixed system.
\end{abstract}

Key words. Galaxy: center - Galaxy: kinematics and dynamics - stars: kinematics - infrared: stars

\section{Introduction}

The dynamical properties of the Galactic centre (GC) star cluster, which hosts the radio source and supermassive black hole (SMBH) Sagittarius A* (Sgr A*), have been subject to intensive research for about two decades. Due to strong interstellar extinction $\left(A_{V} \approx 30\right)$ the GC stars can be observed only in the infrared; most of the work in this field is based on near-infrared (NIR) data ranging from $H$ to $L$ bands (1.5-4 $\mu \mathrm{m}$; see Fig. 1 for an example).

Initially, the central question of this research was whether the GC indeed hosts a central SMBH (e.g. Lynden-Bell \& Rees 1971), which was discussed even before the discovery of the radio point source Sgr A* (Balick \& Brown 1974). Based on statistical arguments using the observed velocity dispersions, it was possible to show in the late 1980s and early 1990s that a central pointlike mass of a few million solar masses was present. Additionally, increasingly better estimates of the distance to the GC became possible (McGinn et al. 1989; Krabbe et al. 1995; Eckart \& Genzel 1997; Ghez et al. 1998; Genzel et al. 1996, 1997, 2000).

* Based on observations at the Very Large Telescope (VLT) of the European Southern Observatory (ESO), Cerro Paranal, Chile.
With improved data quality, especially due to the establishment of speckle imaging and adaptive optics (AO) assisted imaging and spectroscopy, as well as longer observation time lines, more direct tests of the central mass were executed. These efforts led to the observation of Keplerian star orbits in the immediate vicinity $\left(\approx 0.5^{\prime \prime}\right.$ or $\left.\approx 4000 \mathrm{AU}\right)$ of Sgr A* which allowed a direct geometric determination of the mass $M_{\bullet}$ of and the distance $R_{0}$ to the central SMBH (Schödel et al. 2002, 2003; Ghez et al. 2003, 2005, 2008; Eisenhauer et al. 2003a, 2005). Throughout this paper, we adopt a canonical distance $R_{0}=8 \mathrm{kpc}$ and a distance-scaled mass $M_{\bullet}=(4.1 \pm 0.4) \times 10^{6} \times\left(R_{0} / 8 \mathrm{kpc}\right)^{2.3} M_{\odot}$. The scaled mass is taken from Eisenhauer et al. (2005). Initially, Eisenhauer et al. (2005) derived a distance $R_{0}=7.62 \pm 0.32 \mathrm{kpc}$. A recent re-analysis of the data by Gillessen et al. (2008) showed that systematic errors are present in the data which had been neglected in earlier works (also Ghez et al. 2008). In total, the uncertainty on $R_{0}$ is $\approx 0.4 \mathrm{kpc}$ (the $0.32 \mathrm{kpc}$ quoted by Eisenhauer et al. 2005 are the statistical error). We therefore decided to stick to the canonical distance of $8.0 \mathrm{kpc}$. For this $\mathrm{GC}$ distance the image scale is 1 arcsec $\simeq 39 \mathrm{mpc} \simeq 8000 \mathrm{AU}$ in position and $1 \mathrm{mas} / \mathrm{yr} \simeq 38 \mathrm{~km} \mathrm{~s}^{-1}$ in velocity.

In addition to these advances, a dynamically complex structure of the central cluster on scales of $\approx 1-10^{\prime \prime}$ emerged. It 


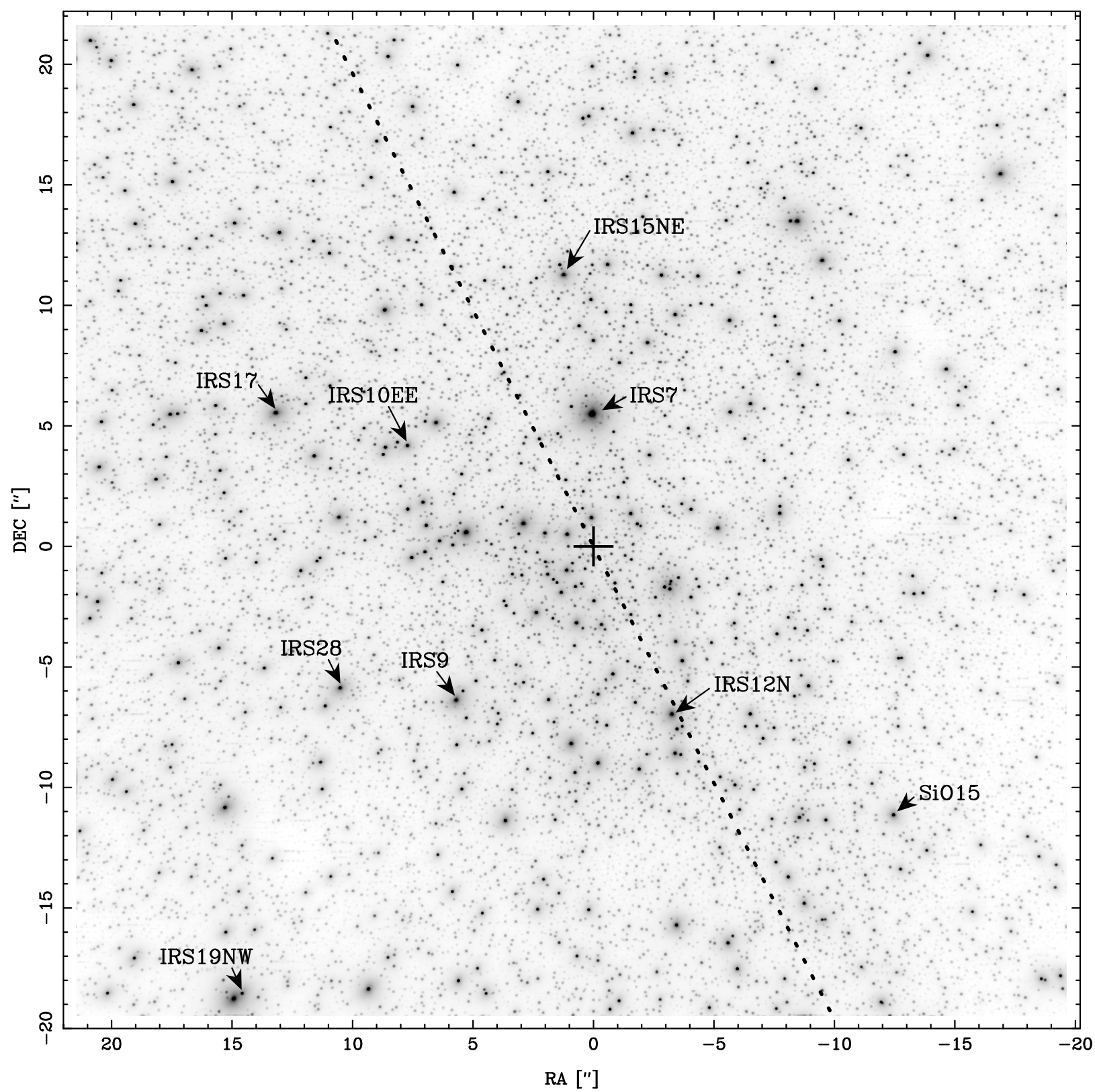

Fig. 1. $K$-band mosaic of the Galactic centre cluster obtained in March 2007; coordinates are RA and Dec in arcsec relative to Sgr A*. This image covers a FOV of $\approx 1.5 \times 1.5 \mathrm{pc}$. The contrast was enhanced by applying unsharp masking. The position of Sgr A* is marked with a cross in the image centre. The dotted line crossing the image indicates the Galactic plane. Stars with names are SiO maser stars used for defining an astrometric reference frame.

was possible to show that the cluster is composed of two main populations: (1) a population of dynamically relaxed, evolved, old (several Gyr), spherically distributed late-type CO absorption line stars; and (2) a relatively small population of young ( $\approx 6 \mathrm{Myr}$ ) OB- and Wolf-Rayet-stars, located in the central arcsecond and in two disks centered on Sgr A* (Genzel et al. 2003; Paumard et al. 2006; Maness et al. 2007).

As most, if not all, nearby galactic systems contain central SMBHs (e.g. Ferrarese \& Ford 2005, and references therein), analyzing the Galactic centre system enhances the understanding of galaxy cores in general. Stellar dynamics is an important tool for the analysis of the central masses (see, e.g., Kormendy \& Bender 1999; Bender et al. 2005, for the case of M 31). In contrast to other galactic nuclei, the centre of the Milky Way can be observed on physical scales small enough to observe accelerations of individual stars within a reasonable (few years) amount of time. Thus the GC is a unique laboratory for studying the dynamical interaction of a SMBH with its immediate stellar environment.

In this article we focus on the properties of the population of evolved late type, $\mathrm{CO}$ absorption stars. We present the as yet most precise kinematical analysis of the central star cluster. This work is based on proper motions and radial velocities extracted from diffraction limited imaging and spectroscopy data obtained from 2002 to 2007.

This paper is organized as follows. In Sect. 2, we summarize the data acquisition and reduction. Section 3 describes the extraction of stellar positions and proper motions from imaging data. Section 4 gives an overview on the collection of line-ofsight velocities from integral-field spectroscopy data. In Sect. 5, we present our findings and discuss them. Section 6 summarizes our results and conclusions. 


\section{Observations and data processing}

\subsection{Imaging}

This work is based on observations with the 8-m-UT4 (Yepun) of the ESO-VLT on Cerro Paranal, Chile. For obtaining imaging data we used the detector system NAOS/CONICA (NACO for short) consisting of the AO system NAOS (Rousset et al. 2003) and the $1024 \times 1024$-pixel NIR camera CONICA (Hartung et al. 2003).

\subsubsection{Data reduction}

We obtained 10 data sets in $H$ and $K$ bands with a pixel scale of 27 mas/pixel (large scale) covering 6 epochs (May 2002, May 2003, June 2004, May 2005, April 2006, March 2007). In this mode each image covers a field of view (FOV) of $28 \times 28^{\prime \prime}$. During each observation the camera pointing was shifted such that the FOV is $\approx 40^{\prime \prime} \times 40^{\prime \prime}$ for a typical data set, centered on Sgr A*.

We executed a much larger number of observations using a smaller pixel scale of 13 mas/pixel (small scale), thus resulting in an image FOV of $14^{\prime \prime} \times 14^{\prime \prime}$ and, again using shifted pointings, typical observation FOVs of $20^{\prime \prime} \times 20^{\prime \prime}$. In total we obtained $42 H$ and $K$ band image sets, 5 to 10 per year with a roughly monthly sampling.

To all images we applied sky-subtraction, bad-pixel and flatfield correction. In order to obtain the best possible signal-tonoise ratios and maximum FOV coverages in single maps, we combined all good-quality images obtained in the same night into mosaics.

\subsubsection{Geometric distortion}

In order to avoid systematic alignment errors when mosaicking single images, we corrected the individual frames for the geometric distortion of the CONICA imager. As there is no publicly available description of the instrumental distortion properties of $\mathrm{NACO}$, we extracted the necessary parameters from our data. We modelled the distortion correction using the radially symmetric standard ansatz

$\mathbf{r}=\mathbf{r}^{\prime}\left(1-\beta \mathbf{r}^{2}\right)$

with

$\mathbf{r}=\mathbf{x}-\mathbf{x}_{C} \quad$ and $\quad \mathbf{r}^{\prime}=\mathbf{x}^{\prime}-\mathbf{x}_{C}$

(e.g. Jähne 2005) $)^{1}$. Here $\mathbf{x}$ and $\mathbf{x}^{\prime}$ are the true and distorted image coordinates respectively, $\beta$ is a parameter describing the strength of the grid curvature, and $\mathbf{x}_{C} \equiv\left(x_{C}, y_{C}\right)$ is the zero point of the distortion on the detector. Details of the modelling procedures are given in the Appendix.

In case of the large scale (27 mas/pixel) images we found

$x_{C} \simeq 577 \ldots 629$ pixels

$y_{C} \simeq 775 \ldots 823$ pixels

$\beta \simeq 2.97 \ldots 3.40 \times 10^{-9}$ pixels $^{-2}$.

1 See also the electronic manual of the public Gemini North Galactic Center Demonstration Science Data Set for an application on GC imaging data.
For the small scale (13 mas/pixel) images the parameters were $x_{C} \simeq 573 \ldots 839$ pixels

$y_{C} \simeq 629 \ldots 948$ pixels

$\beta \simeq 2.06 \ldots 13.27 \times 10^{-10}$ pixels $^{-2}$.

For the small scale images the distortion is marginally significant and therefore hard to measure, as evident in the wide range of values found for the curvature $\beta$. Nevertheless, it is possible to state qualitatively that for the large scale images $(\beta \approx 3 \times$ $10^{-9}$ pixels $\left.^{-2}\right)$ the distortion is clearly stronger than for the small scale images $\left(\beta \approx 5 \times 10^{-10}\right.$ pixels $\left.^{-2}\right)$.

\subsubsection{Image registration and mosaicking}

After extracting the distortion parameters, we registered all single frames with respect to a common coordinate grid to ensure alignment to sub-pixel accuracy. After correcting for geometric distortion, no systematic effects of spatial higher-order should remain. In such a case, the image registration can be described by spatial first-order transformations

$x^{\prime}=a_{0}+a_{1} x+a_{2} y$

$y^{\prime}=b_{0}+b_{1} x+b_{2} y$

which cover translations, rotations, scalings, and shears.

The image registration process consists of two main steps. Firstly, we define one master image (usually the first image of a set) as a zero point. We pick a set of bright stars with coordinates $\left\{\mathbf{x}_{\text {ref }}^{0}\right\}$ serving as reference.

Secondly, we find for each image the transformation $T_{m}$ which transforms the coordinates of the reference stars measured in image $m$ to the coordinates measured in the zero image:

$T_{m}:\left\{\mathbf{x}_{\text {ref }}^{m}\right\} \longrightarrow\left\{\mathbf{x}_{\text {ref }}^{0}\right\}$.

The final mosaic results from computing for each pixel in image $m$ its new position in the mosaic grid (corresponding to image 0 ) by applying $T_{m}$. For the transformations we solved the overdetermined sets of equations by means of least-squares fits. We used the routines by Montenbruck \& Pfleger (1989) implemented in the MPE data analysis software package DPUSER ${ }^{2}$. For each image, the flux values of its pixels were then interpolated to the corresponding mosaic pixels.

Comparing the typical residual alignment errors before and after distortion correction and registration (Fig. 2) shows clearly the improvement in mosaic quality. While simple shift-and-add leads to inaccuracies as high as some tenths of a pixel, the distortion correction alone provides a strong improvement. When taking into account the grid curvature only, systematic first-order effects (shifts, rotations, and shear) are left. After registering the images, the typical residual errors (pairwise between image overlap areas) are of the order $\approx 0.05$ pixels, corresponding to $\approx 1.4$ mas (large scale) and $\approx 0.7$ mas (small scale), respectively.

\subsection{Spectroscopy}

For obtaining spectroscopic data we used SINFONI, a combination of the integral field spectrometer SPIFFI (Eisenhauer et al. $2003 \mathrm{~b}, \mathrm{c}$ ) and the adaptive optics system MACAO (Bonnet et al. 2003, 2004).

\footnotetext{
${ }^{2}$ Developed by Thomas Ott; http://Www.mpe.mpg.de/ ott/ dpuser/history.html
} 

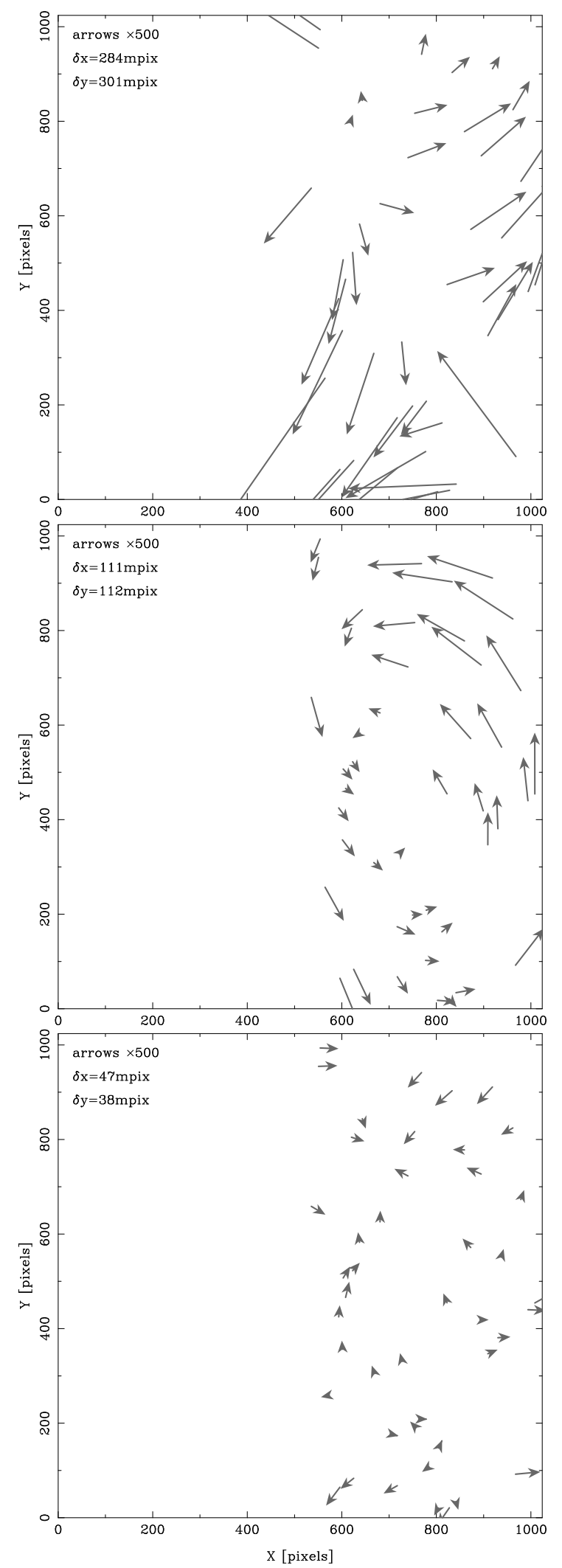

Fig. 2. Residual image alignment errors before and after distortion correction and image registration, comparing the overlap area (right hand half of the first image of the data set) of two 27 mas/pixel scale $K$ band images obtained in March 2007. Arrows mark absolute values ( 1 unit $=$ 2 milli-pixels) and directions of residuals, $\delta x(\delta y)$ are the rms of residuals in $x(y)$. Top panel: sub-pixel accurate shift-and-add only. Central panel: after correcting for geometric distortion, before registration. Bottom panel: after distortion correction and registration.
SINFONI's data output consists of cubes. The cubes have two spatial axes with dimensions of 64 and 32 pixels respectively and one spectral axis of 2048 pixels length.

Depending on the plate scale, individual cubes covered regions of $0.8 \times 0.8^{\prime \prime}, 3.2 \times 3.2^{\prime \prime}$, or $8 \times 8^{\prime \prime}$; the latter was used in seeing-limited mode only. The spectra covered either the $K$ band (with a spectral resolution of $R=4500$ ) or the band range $H+K$ $(R=2500)$.

SINFONI shows a substantial geometric distortion. This distortion can be parametrized as a 2-dimensional second-order polynomial. We applied the publicly available distortion parameters for correction of our data.

After sky subtraction, bad-pixel-, and flat-field-correction, the wavelength scale was calibrated. This calibration used emission line gas lamps and was finetuned on the atmospheric $\mathrm{OH}$ lines. Atmospheric absorption features were removed by dividing by the spectrum of a calibration star.

\section{Astrometry}

\subsection{Source selection}

In order to determine positions and proper motions for as many stars as possible, we first constructed a source list from a highquality large scale $\mathrm{K}$ band mosaic with $\approx 40 \times 40^{\prime \prime} \mathrm{FOV}$ obtained in May 2005 (epoch 2005.36). In this image we identified and listed all stars above a given significance threshold using the algorithm FIND (Stetson 1987). This algorithm searches an image for positive brightness perturbations and identifies them as stars, if their sharpness and roundness parameters are located within given limits.

Out of the list of all detected sources we excluded those overlapping (i.e. separated by less than $\approx 2 F W H M \mathrm{~s} / \approx 130$ mas) with neighbouring stars and thus unusable for precise astrometry. The list of remaining "good" stars contains 6037 objects down to magnitudes of $K \approx 18$. For all sources the diffraction-limited cores were fit as 2-dimensional elliptical Gaussian brightness distributions.

This procedure of source selection leaves us with a spatial source distribution which is very different from the physical stellar surface density. Due to the restrictions outlined in the previous paragraphs, the sample distribution shows an approximately constant surface density $\left(\approx 4\right.$ sources/arcsec ${ }^{2}$ ) across the entire FOV. This does not harm the analysis of kinematic parameters (velocities, velocity dispersions) because there is no bias in velocities. However, in the later stages of this analysis we analyse spatially de-projected parameters such as 3D dispersions. As the outcome of this de-projection crucially depends on the assumed 3D source distribution, we make use of earlier analyses of the 2D and 3D star distributions (Genzel et al. 1996, 2000, 2003; Mouawad et al. 2005; Schödel et al. 2007).

In each individual mosaic $n$ the master list stars were re-identified and their detector positions $\left\{\mathbf{X}^{n}\right\}$ were fit with 2-dimensional elliptical Gaussian profiles. The formal detector position accuracies were typically (mode of histogram) $\approx 0.025$ pixels (per coordinate) in both plate scales, i.e. $\approx 0.68(0.33)$ mas in the large (small) plate scale.

\subsection{Astrometric coordinates}

In order to convert the image positions of the source list stars into absolute astrometric coordinates, we initially use a reference set of $9 \mathrm{SiO}$ maser stars located in the FOV. For these stars absolute positions and motions are known from radio observations 

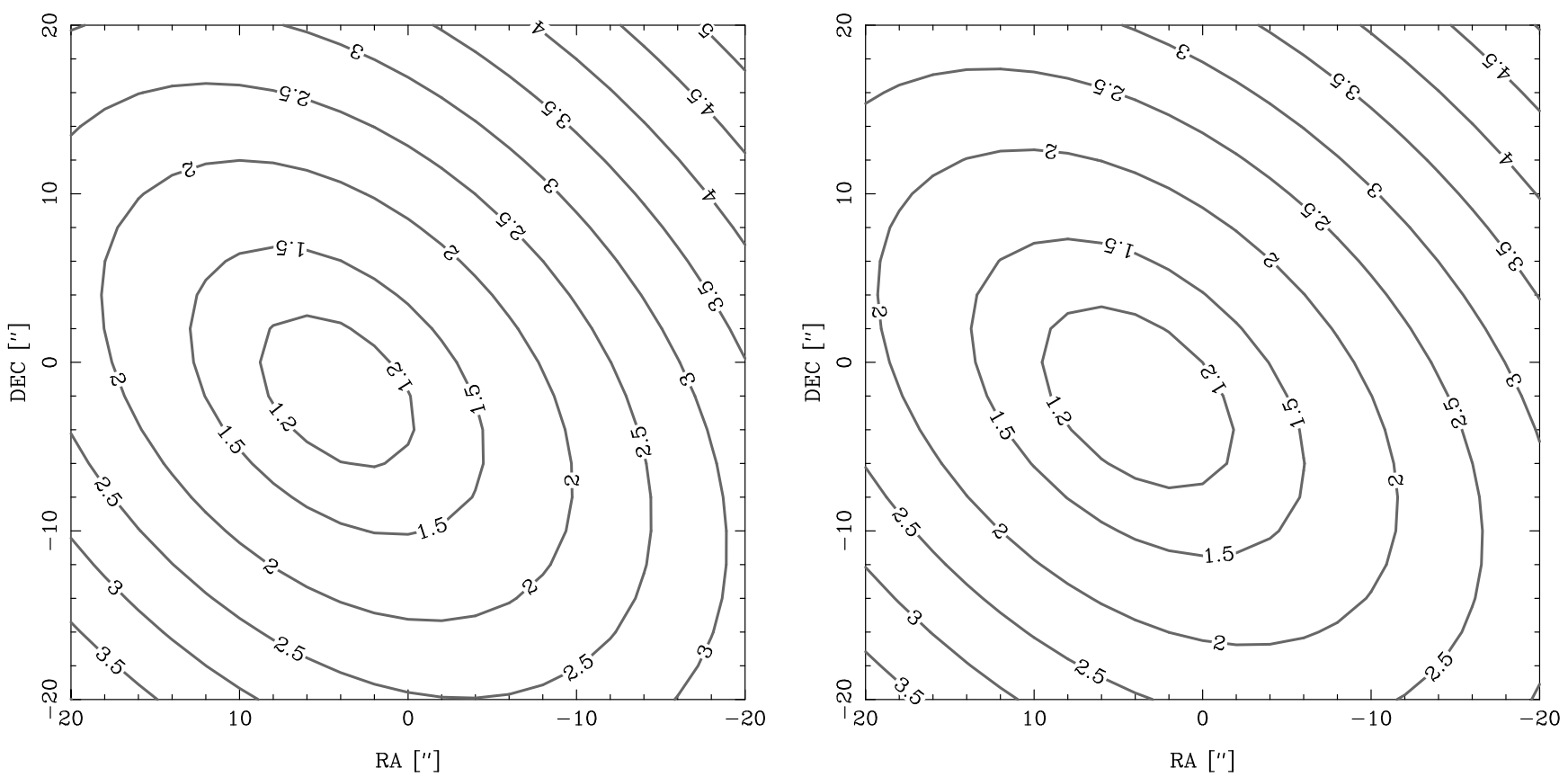

Fig. 3. Statistical uncertainty of absolute radio reference frame coordinates as a function of position due to transformation errors, separately for RA (left hand panel) and Dec (right hand panel). The contour values are given in units of milli-arcseconds. Across the field of view the errors vary in the range $1.2 \ldots 5$ mas. The contours mirror the alignment of the reference stars in the sky plane (see Fig. 1).

(Reid et al. 2007). They are bright sources in both radio and NIR, and are therefore well-suited for cross-calibration.

Although there are no simultaneous observations in the two wavelength regimes, there are radio position measurements from both before and after the epoch of the NIR reference image. The astrometric positions of the $\mathrm{SiO}$ maser stars at epoch 2005.36, $\left\{\mathbf{x}_{\text {maser }}^{0}\right\}$, can then be obtained by interpolation (see Reid et al. 2007 for details). Using the NIR image detector positions $\left\{\mathbf{X}_{\text {maser }}^{0}\right\}$, we find a transformation

$T_{A}:\left\{\mathbf{X}_{\text {maser }}^{0}\right\} \longrightarrow\left\{\mathbf{x}_{\text {maser }}^{0}\right\}$.

By applying $T_{A}$, we calculate astrometric (reference epoch) positions $\left\{\mathbf{x}^{0}\right\}$ for all 6037 stars:

$\left\{\mathbf{X}^{0}\right\} \longrightarrow T_{A}\left(\left\{\mathbf{X}^{0}\right\}\right)=\left\{\mathbf{x}^{0}\right\}$.

The absolute accuracies of these coordinates vary in the range $1.2 \ldots 5$ mas depending on position (see Fig. 3 for a contour map); details can be found in the Appendix.

Unfortunately, in our NIR data no absolute astrometric reference source is available and the $\mathrm{SiO}$ maser stars are present only in some of the large scale (27 mas/pix) images. We therefore defined a relative astrometric reference frame tied to an ensemble of $\approx 560$ well-behaved (meaning bright and well separated from neighbouring sources) stars with astrometric positions $\left\{\mathbf{x}_{\mathrm{ref}}^{0}\right\} \subset\left\{\mathbf{x}^{0}\right\}$. For each image $n$ we use the corresponding detector positions $\left\{\mathbf{X}_{\text {ref }}^{n}\right\}$ to compute a linear transformation

$T_{n}:\left\{\mathbf{X}_{\text {ref }}^{n}\right\} \longrightarrow\left\{\mathbf{x}_{\text {ref }}^{0}\right\}$.

In this step we assume that our reference star ensemble $\left\{\mathbf{x}_{\text {ref }}^{0}\right\}$ is at rest in average, i.e. $\left\langle\left\{\mathbf{x}_{\text {ref }}^{0}\right\}\right\rangle=\left\langle\left\{\mathbf{x}_{\text {ref }}^{n}\right\}\right\rangle$. We note that our procedure is very similar to the local-transformation approach outlined by Anderson et al. (2006). The transformation $T_{n}$ is used to calculate the astrometric positions of all stars via

$\left\{\mathbf{X}^{n}\right\} \longrightarrow T_{n}\left(\left\{\mathbf{X}^{n}\right\}\right)=\left\{\mathbf{x}^{n}\right\}$.
In the small scale (13 mas/pix) images, typically only $\approx 100$ of the reference stars are present. This means that we only have relatively small subsets $\left\{\mathbf{X}_{\text {small }}^{n}\right\} \subset\left\{\mathbf{X}_{\text {ref }}^{n}\right\}$ at hand for calibration. Therefore we first analyse the large scale (27 mas/pix) images and compute proper motions for all reference stars (see also Sect. 3.3). With the proper motions at hand, we compute for each image $n$ the expected astrometric positions $\left\{\mathbf{x}_{\text {small }}^{n}\right\}$ via linear interpolation. From this we find the (1st order) transformation

$T_{n^{\prime}}:\left\{\mathbf{X}_{\text {small }}^{n}\right\} \longrightarrow\left\{\mathbf{x}_{\text {small }}^{n}\right\}$

which leads to the astrometric positions of all stars via

$\left\{\mathbf{X}^{n}\right\} \longrightarrow T_{n^{\prime}}\left(\left\{\mathbf{X}^{n}\right\}\right)=\left\{\mathbf{x}^{n}\right\}$.

This procedure ensures that the small scale images are tied to the reference frame of the large scale images.

\subsection{Proper motions}

We computed stellar proper motions $\mathbf{v}_{x y}$ by fitting linear functions to astrometric star positions $\mathbf{x}$ vs. time $t$ :

$\mathbf{x}(t)=\mathbf{v}_{x y} t+\mathbf{x}(0)$

In order to determine proper errors for the stellar velocities, we applied outlier rejection and error rescaling to the data. The typical (mode of histogram) measurement error of a star position is $\approx 0.9$ mas for both image scales. The timeline of observations is five years. The number of epochs is 10 for the large (27 mas/pix) scale and 42 for the small (13 mas/pix) scale imaging data.

In total we were able to extract proper motions for 5548 stars located in the large scale fields; out of these, 755 sources were additionally covered by the small scale images. Typical (mode of histogram) statistical proper motion accuracies are $\approx 0.18 \mathrm{mas} / \mathrm{yr}$ per coordinate for the large scale data sets and $\approx 0.1 \mathrm{mas} / \mathrm{yr}$ 

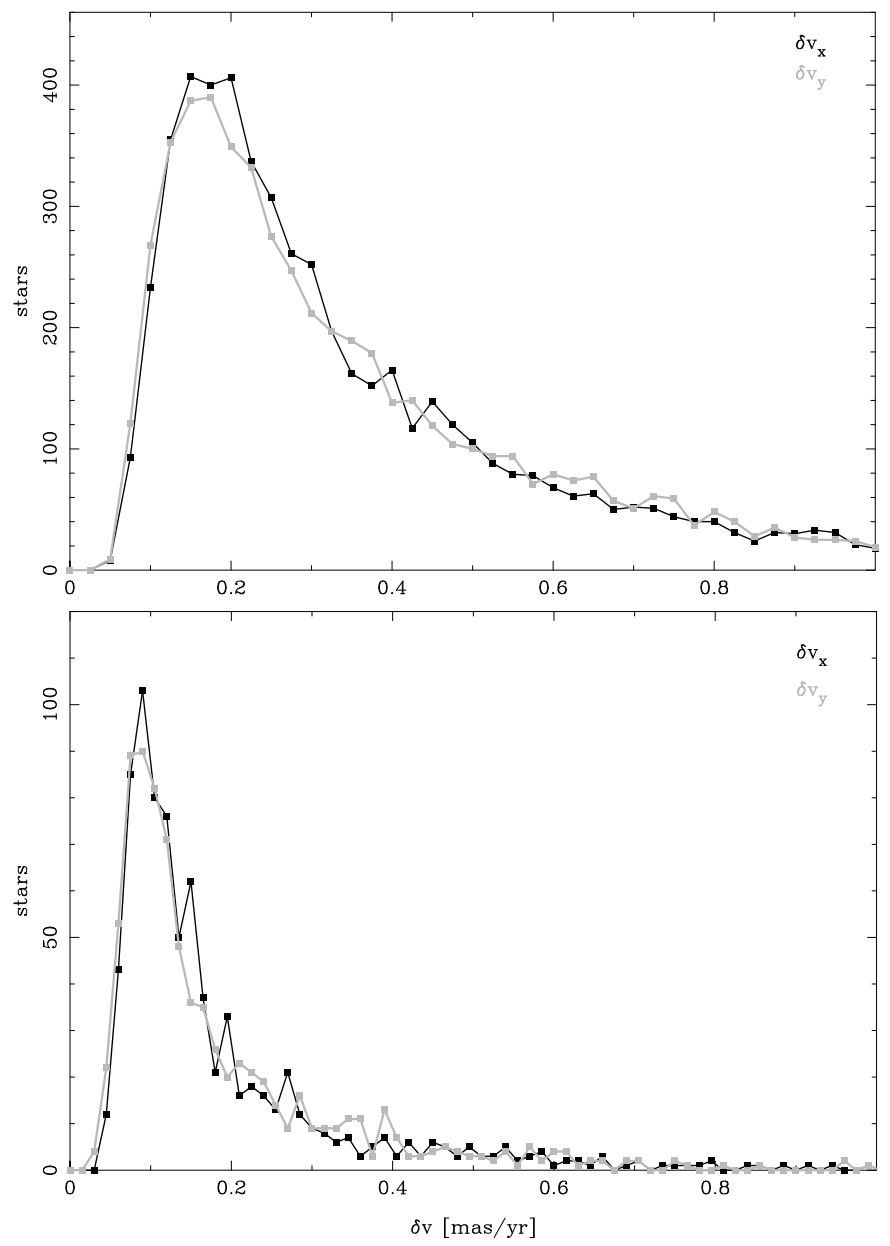

Fig. 4. Histograms of proper motion errors. Top panel: large pixel scale (27 mas/pix). Bottom panel: small pixel scale (13 mas/yr). The distributions peak at $\approx 0.18 \mathrm{mas} / \mathrm{yr}$ in case of the large pixel scale and at $\approx 0.1 \mathrm{mas} / \mathrm{yr}$ in case of the small pixel scale, corresponding to $6.8 \mathrm{~km} \mathrm{~s}^{-1}$ and $3.8 \mathrm{~km} \mathrm{~s}^{-1}$ respectively.

per coordinate for the small scale fields. This corresponds to $\approx 6.8 \mathrm{~km} \mathrm{~s}^{-1}$ and $\approx 3.8 \mathrm{~km} \mathrm{~s}^{-1}$, respectively. The error distributions are presented in Fig. 4.

We focused on the behaviour of the late-type population of stars which is expected to be dynamically relaxed (Genzel et al. 2003). Therefore we excluded 103 spectroscopically identified early-type stars which are known to mainly move in disks (Paumard et al. 2006); this reduced the number of 2D velocity stars from 5548 to 5445 . One should however note that probably some more, so far unidentified, early-type stars are still included in our proper motion sample (under investigation by Bartko et al., in prep.).

An additional, systematic uncertainty is introduced by the relative astrometric reference frame. This frame is based on stars with proper motions known only a posteriori and with respect to the star cluster. Thus a systematic motion of the reference frame is possible. Using the average number of applicable large scale image reference stars, which is 433 , and the rms velocity of the reference stars ( $3.6 \mathrm{mas} / \mathrm{yr}$ or $137 \mathrm{~km} \mathrm{~s}^{-1}$ ), we estimate this systematic uncertainty (standard error) to be $0.17 \mathrm{mas} / \mathrm{yr}$ or $6.4 \mathrm{~km} \mathrm{~s}^{-1}$.

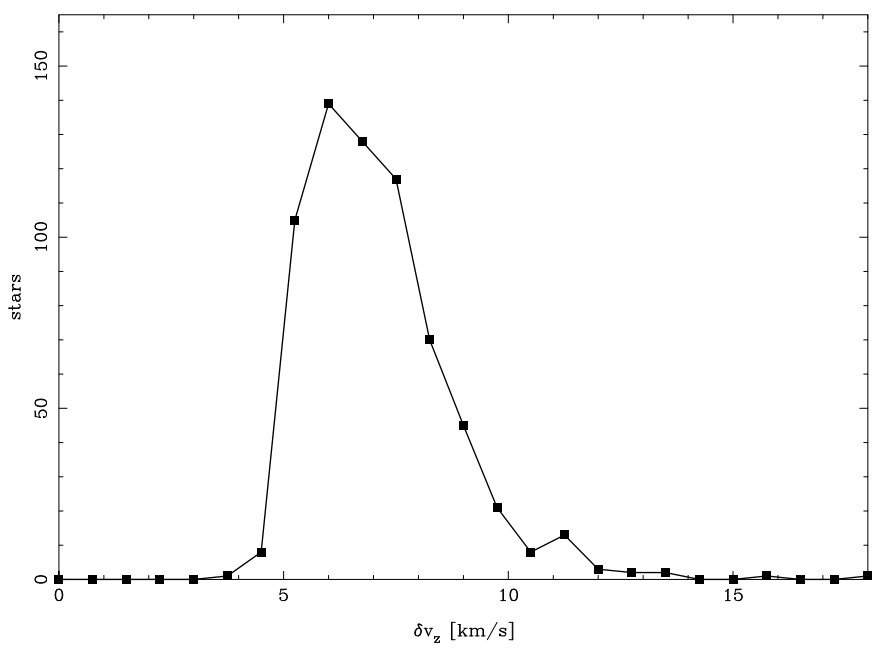

Fig. 5. Histogram of statistical errors in line-of-sight velocity for 664 stars. The distribution peaks at $\approx 7 \mathrm{~km} \mathrm{~s}^{-1}$.

\section{Radial velocities}

\subsection{Source selection}

We extracted spectra of late-type $\mathrm{CO}$ absorption stars from the SINFONI cubes. For each star we selected source and background pixels by hand. Background pixels were selected from pixels surrounding the source pixels. A corrected star spectrum results from subtracting the average of the background pixels from the average of the source pixels. This operation is necessary in order to take into account incomplete sky subtraction, nebular contamination, and flux spillover from neighbouring sources.

Due to the small FOV of SINFONI $\left(8^{\prime \prime} \times 8^{\prime \prime}\right.$ at most), our target area (central $\approx 40^{\prime \prime} \times 40^{\prime \prime}$ ) is covered only partially; see Paumard et al. (2006) for a detailed overview, especially their Fig. 1. Additionally, the various data sets show different pixel scales, Strehl ratios, and photometric completenesses. This incomplete coverage of the star cluster is a serious limit for $3 \mathrm{D}$ de-projection and kinematic phase-space analysis of the data.

\subsection{Velocity fitting}

We extracted stellar radial velocities by correlating the observed spectra with a theoretical template spectrum (e.g. Tonry \& Davis 1979). The model spectrum of a CO star obtained from the MARCS stellar model-atmosphere and flux library (Gustafsson et al. 2003) served as template. Main model parameters were temperature $T_{\text {eff }}=4250 \mathrm{~K}$, gravitational acceleration $\log g=$ $0\left[\mathrm{~cm} / \mathrm{s}^{2}\right]$, micro-turbulence velocity $v_{\text {turb }}=2 \mathrm{~km} \mathrm{~s}^{-1}$, and solar metallicities. The model parameters - especially $T_{\text {eff }}-$ were selected in order to fit the most numerous stars in our sample, which are red clump stars (Maness et al. 2007).

As we were especially interested in the behaviour of evolved late-type stars, we focused our analysis on the CO bandhead lines in the wavelength range $\approx 2.28 \ldots 2.37 \mu \mathrm{m}$. If the maximum correlation was lower than 0.55 , the computed velocity was rejected as unreliable. We chose this threshold experimentally after checking the spectra by eye. All velocities were corrected to the local standard of rest using the standard IAU solar motion (see, e.g., Kerr \& Lynden-Bell 1986, and references therein).

In total we extracted radial velocities for 664 late-type stars. Typical statistical velocity accuracies are $\approx 7 \mathrm{~km} \mathrm{~s}^{-1}$; their distribution is presented in Fig. 5. Additionally, a systematic uncertainty is introduced by the selection of the model spectrum 


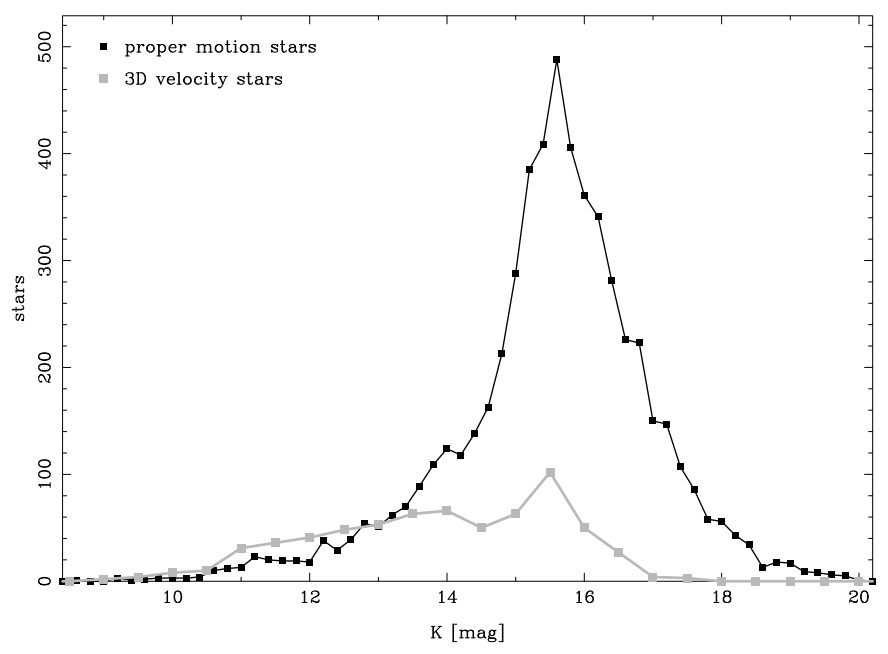

Fig. 6. $K$ magnitude distributions of all 5445 (non early-type) proper motion stars (black curve) and the 664 3D velocity stars (grey curve). Please note the different binnings. The distributions peak in the range $K \approx 15 . . .16$; this is the regime of the Red Clump stars. For $K>16$, the completeness quickly decreases.

parameters. From a comparison of several model spectra, we estimate this systematic error to be $\approx 5 \mathrm{~km} \mathrm{~s}^{-1}$. Figure 6 shows the $K$ magnitude distribution of the 5445 proper motion stars and the 664 stars with radial velocities.

\section{Results and discussion}

\subsection{Isotropy and rotation}

With proper motions for 5445 stars and 3D-velocity vectors for 664 stars, we extracted the dynamical properties of the cluster. Our data provide information for projected distances from Sgr A* up to about 27 arcsec. As a first step we computed velocity dispersions along all coordinate directions.

In order to calculate these parameters we used the method by Hargreaves et al. (1994). For each coordinate axis $q$, this algorithm computes the average velocity $\left\langle v_{q}\right\rangle$ and the velocity dispersion $\sigma_{q}$ for a given ensemble of stars using the iterative scheme

$\left\langle v_{q}\right\rangle=\frac{\sum_{i} w_{i} \cdot v_{q, i}}{\sum_{i} w_{i}}$

$\sigma_{q}^{2}=\frac{\sum_{i}\left[\left(v_{q, i}-\left\langle v_{q}\right\rangle\right)^{2}-\delta_{q, i}^{2}\right] \cdot w_{q, i}^{2}}{\sum_{i} w_{q, i}^{2}}$

with $v_{q, i}$ being the $q$ component of the velocity of star $i, \delta_{q, i}$ being the respective error, and $w_{q, i}=1 /\left(\delta_{q, i}^{2}+\sigma_{q}^{2}\right)$ being the star's weight. In general, not more than three iterations are necessary to obtain stable results; we usually used five. The respective statistical errors are

$\delta\left\langle v_{q}\right\rangle=\sqrt{\frac{\sigma_{q}^{2}+\left\langle\delta_{q, i}^{2}\right\rangle}{N}}$

$\delta \sigma_{q}=\frac{\sigma_{q}^{2}+\left\langle\delta_{q, i}^{2}\right\rangle}{\sigma_{q} \sqrt{2 N}}$.

$N$ is the number of stars, $\left\langle\delta_{q, i}^{2}\right\rangle$ is the mean squared statistical velocity error per star. For the case $\left\langle\delta_{q, i}^{2}\right\rangle \ll \sigma_{q}^{2}$ this simplifies to $\delta\left\langle v_{q}\right\rangle \simeq \sigma_{q} / \sqrt{N}$ and $\delta \sigma_{q} \simeq \sigma_{q} / \sqrt{2 N}$.

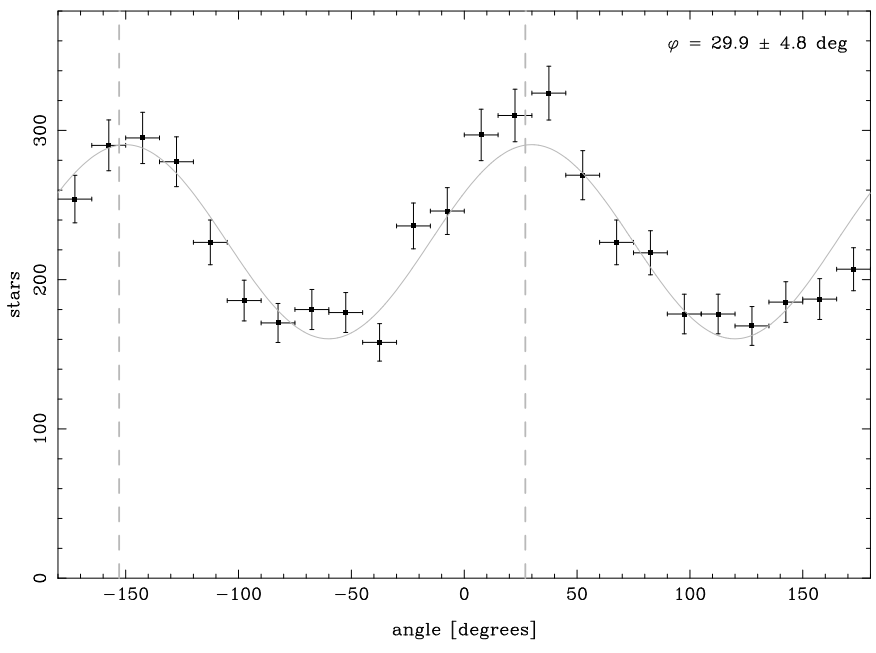

Fig. 7. Modulations in stellar proper motions. Shown here is the number of stars vs. angle of the proper motion vectors. The angle is defined as atan $2\left(v_{x}, v_{y}\right)$ and counted from north to east. Black points with error bars are the data; horizontal error bars mark the full bin widths, vertical error bars are Poisson $\sqrt{N}$ errors. This diagram tests the preferential orientations of proper motion vectors on sky. The vertical dashed grey lines mark the location of the Galactic plane $\left(+27.1^{\circ}\right.$ and counter-direction). A cosine fit to the data (grey curve) finds a phase $\phi=+30 \pm 5^{\circ}$, in agreement with the orientation of the Galaxy.

For the two velocity dispersions in RA (labelled $x$ ) and Dec (labelled $y$ ) using all 5445 proper motion stars we found the values

$\sigma_{x}=2.668 \pm 0.027 \mathrm{mas} / \mathrm{yr}$

$\sigma_{y}=2.824 \pm 0.028 \mathrm{mas} / \mathrm{yr}$

implying that the dispersions in RA and Dec are signicantly different (by about $4 \sigma$ ).

To check the amount and geometric structure of a possible anisotropy in the proper motion vectors we tested their preferential orientations on sky. For each star we computed the angle $\psi=$ $\operatorname{atan} 2\left(v_{x}, v_{y}\right)$ which is counted from north to east ${ }^{3}$. The resulting histogram is shown in Fig. 7. In case of isotropy the distribution would be flat. The histogram, however, shows a highly significant cosine-like pattern. This pattern is consistent with the signature of a rotating disk seen edge-on, but also with an intrinsic anisotropy in random motions. Fitting this pattern with a cosine profile reveals a phase of $+30 \pm 5^{\circ}$, which is in agreement with the plane of the Milky Way located at $+27.1^{\circ}$ (J2000).

The distribution shown in Fig. 7 shows the presence and orientation of a rotation and/or anisotropy pattern, but not its strength in terms of velocities or velocity dispersions. In order to quantify the modulation of the proper motion distribution, we used the following ansatz: for a given principal coordinate axis we computed the velocity dispersions parallel $\left(\sigma_{\|}\right)$and perpendicular $\left(\sigma_{\perp}\right)$ to this axis using all available proper motions. Then we calculated the difference in squares of these two dispersions, $\Delta \sigma^{2}=\sigma_{\|}^{2}-\sigma_{\perp}^{2}$. By rotating the principal axis stepwise on sky, we obtained $\Delta \sigma^{2}$ as a function of the angle. The resulting curve, here using a step size of $5^{\circ}$, is shown in the top panel of Fig. 8. Since for all data points the same set of proper motions is used, the points are correlated. Using a cosine fit to describe the data, we find an amplitude of $\Delta \sigma_{\max }^{2}=2.00 \pm 0.21(\mathrm{mas} / \mathrm{yr})^{2}$.

\footnotetext{
${ }^{3}$ Where atan2 is the quadrant-preserving arctangent.
} 

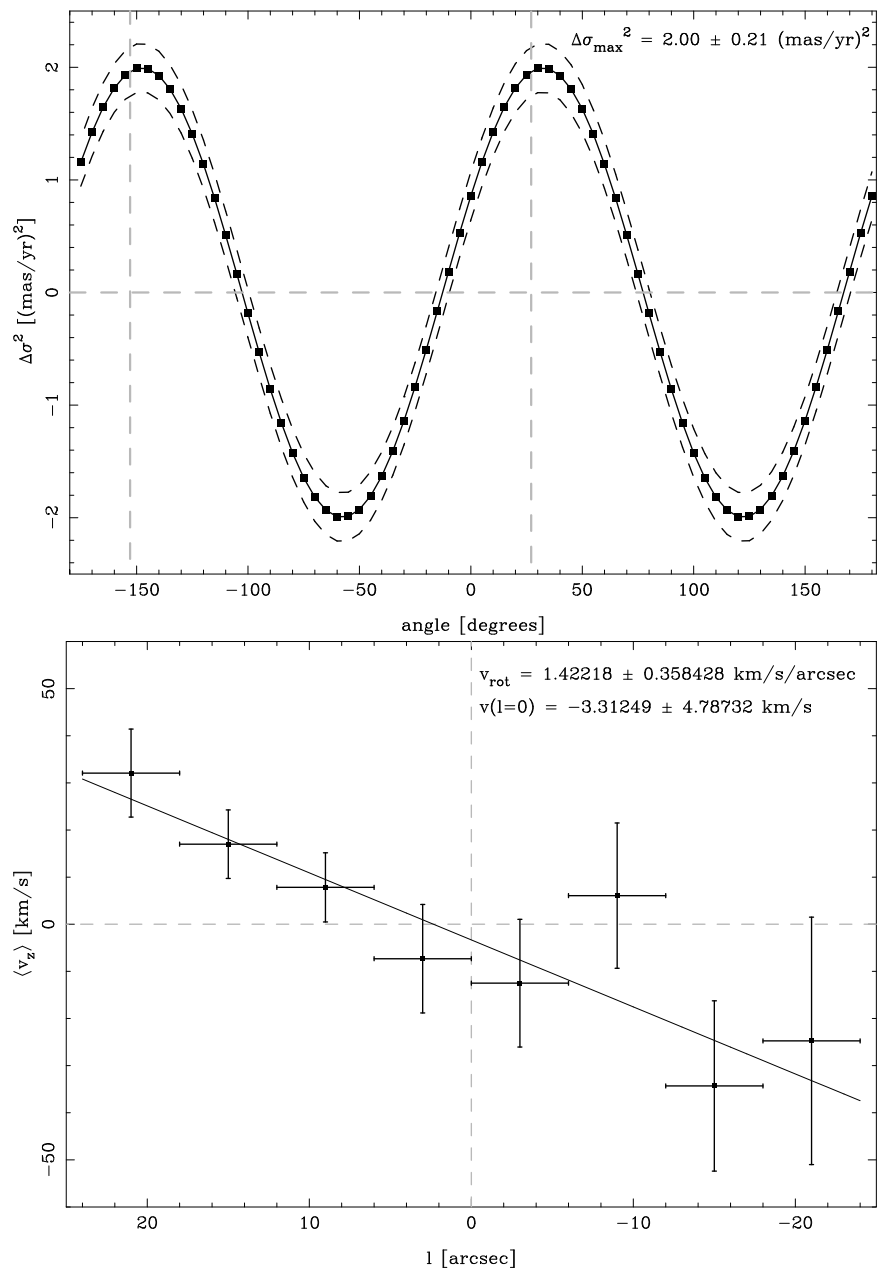

Fig. 8. Signatures of rotation and/or anisotropy in proper motions and radial velocities. Top panel: angle on sky (counted from $\mathrm{N}$ to $\mathrm{E}$ ) vs. difference in square dispersions $\Delta \sigma^{2}=\sigma_{\|}^{2}-\sigma_{\perp}^{2}$. Here $\sigma_{\|}\left(\sigma_{\perp}\right)$ is the velocity dispersion parallel (perpendicular) to a given principal axis which is rotated stepwise. The black dots and the black curve show the observed modulation, dashed black curves mark the 1- $\sigma$ uncertainty range. Vertical grey dashed lines mark the position of the Galactic plane, the horizontal grey dashed line is the zero level of $\Delta \sigma^{2}$. The modulation has an amplitude of $\Delta \sigma_{\max }^{2}=2.00 \pm 0.21(\mathrm{mas} / \mathrm{yr})^{2}$. Bottom panel: average radial velocities $\left\langle v_{z}\right\rangle$ vs. Galactic longitude $l$. Black points are the data, error bars along the $l$ axis mark the full bin sizes, error bars in velocity direction are 1- $\sigma$-errors. Grey dashed lines mark the zero levels of $l$ and $\left\langle v_{z}\right\rangle$. As the number of stars is smaller for $l<0$, errors are larger in this range. A linear fit to the data (continuous black line) obtains a rotation velocity of $1.4 \pm 0.4 \mathrm{~km} \mathrm{~s}^{-1} /$ arcsec.

As shown above, the geometry of the cluster kinematics is in good agreement with the orientation of the Galaxy. Thus relative Galactic coordinates $l, b$ are a more natural coordinate system than ecliptic coordinates $\alpha, \delta$. In the following discussion we will therefore preferentially focus on coordinates and velocities transformed into relative Galactic coordinates, using $(l, b)_{\text {Sgr A* }}=(0,0)$.

In stellar line-of-sight velocities, rotation in the sense of general Galactic rotation was previously reported (McGinn et al. 1989; Genzel et al. 1996, and references therein). Therefore we computed for our 664 radial velocity stars (which form a subset of the proper motion stars) the average stellar radial velocities in given $l$ bins. The resulting pattern is shown in the bottom panel of Fig. 8. Using a linear fit to describe the data points, we find the velocities to be zero within the errors $\left(4.8 \mathrm{~km} \mathrm{~s}^{-1}\right)$ at $l=0$,

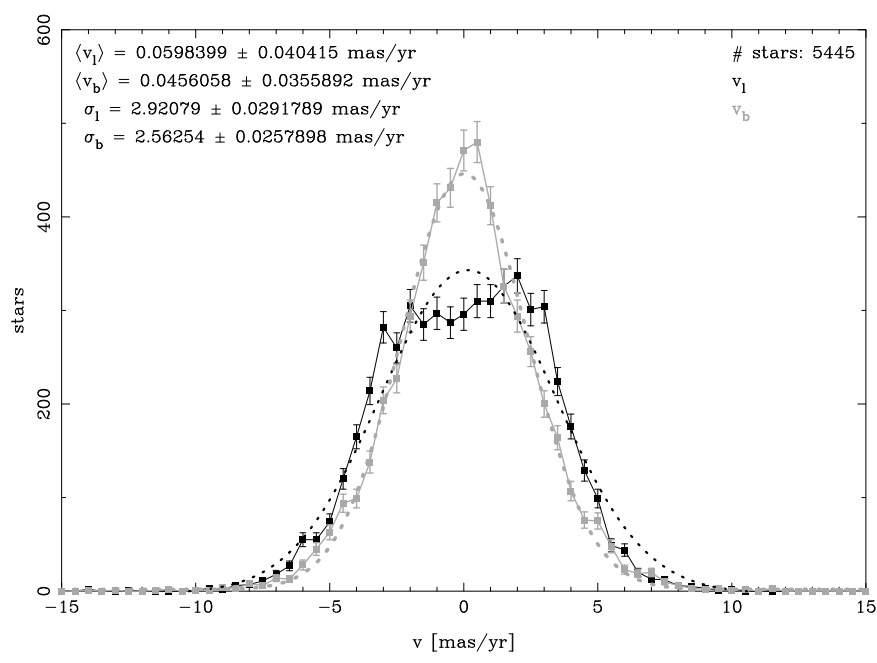

Fig. 9. Histograms of proper motions in $l$ and $b$ of all proper motion stars. Error bars mark the Poisson errors. Dotted lines represent the respective best-fitting Gaussians. Average velocities $\left\langle v_{l, b}\right\rangle$ and velocity dispersions $\sigma_{l, b}$ are given in the plot. Whereas the $b$ velocities appear to be normally distributed, the $l$ velocities show a clear rotation pattern.

positive (i.e. receding from the observer) towards positive Galactic longitudes, and negative (i.e. approaching the observer) towards negative $l$, as expected. This does however not imply that the physical (projected) rotation profile actually follows a linear relation; given the limited accuracies of the data, using a more complex rotation model is not justified. Keeping this in mind, we find a rotation velocity of $1.42 \pm 0.36 \mathrm{~km} \mathrm{~s}^{-1} / \operatorname{arcsec}$. This corresponds to a $4-\sigma$-detection of the Galactic rotation in radial velocities for $|l| \leq 24$ arcsec.

The velocity distributions in $l$ and $b$ for all 5445 proper motion stars are shown in Fig. 9 together with the respective bestfitting Gaussian profiles. The velocities in $b$ appear to be normally distributed. In contrast, the histogram of the $l$ velocities shows clear broadening and flattening. The pattern can be approximately described as a convolution of a Gaussian with width $\sigma_{b}$ and two $\delta$-peaks located at roughly $\pm 2.5 \mathrm{mas} / \mathrm{yr}$. This corresponds to the edge-on view through a system rotating with a fixed rotation velocity of $\approx 2.5$ mas/yr. However, this number is an averaged and projected value and affected by the finite FOV; therefore it must not be read as the physical rotation velocity. We will discuss this quantitatively in Sect. 5.3.

\subsection{Distribution of stellar $3 D$ speeds}

Analysing the distribution of the stars' $3 \mathrm{D}$ speeds $v_{3 \mathrm{D}}$ allows one to test wheteher the cluster is dynamically relaxed. In case of the nuclear cluster, the velocity dispersion scales with the projected distance from Sgr A*, $r$, like $\sigma \propto r^{-0.5}$. As we do not have a detailed dynamical model at hand, we approximate the expected $3 \mathrm{D}$ speed distribution as a superposition of local (meaning in $r$ bins) Maxwellian distributions.

In order to test the distribution of the GC star speeds, we analysed 664 stars with known 3D velocities. For each star we computed a bias-corrected ${ }^{4} 3 \mathrm{D}$ speed

$v_{3 \mathrm{D}}=\sqrt{v_{x}^{2}+v_{y}^{2}+v_{z}^{2}-\delta v_{x}^{2}-\delta v_{y}^{2}-\delta v_{z}^{2}}$

\footnotetext{
4 Velocity squares are limited to values $\geq 0$. Therefore the statistical velocity errors systematically shift the results towards higher values.
} 


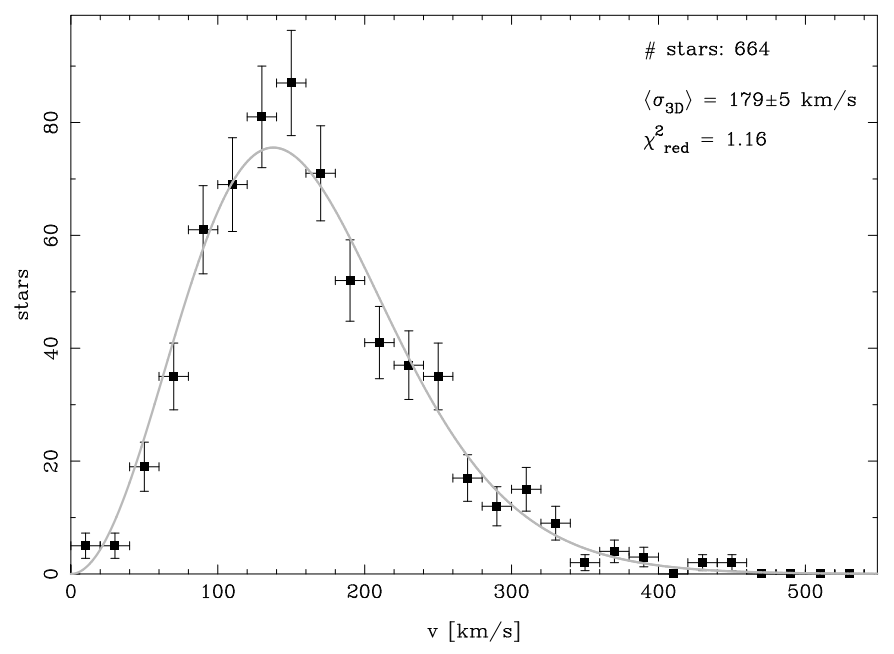

Fig. 10. Distribution of stellar speeds for all 3D velocity stars. Points with error bars are data; errors in star numbers are Poisson errors, errors in $v$ mark the full bin widths. The continuous grey line corresponds to a superposition of Maxwellian distributions for local velocity dispersions $\sigma_{3 \mathrm{D}}(r)$; this line is not a fit to the data. Observed and model distributions are in good agreement; we find $\chi_{\text {red }}^{2}=1.16$.

where $v_{x, y, z}$ are the velocities (in $\mathrm{km} \mathrm{s}^{-1}$, assuming $R_{0}=8 \mathrm{kpc}$; see Sect. 1, 5.5) and $\delta v_{x, y, z}$ are the respective statistical errors.

In a separate step, we calculated $3 \mathrm{D}$ velocity dispersions via

$\sigma_{3 \mathrm{D}}(r)=\sqrt{\sigma_{x}^{2}(r)+\sigma_{y}^{2}(r)+\sigma_{z}^{2}(r)}$.

This $\sigma_{3 \mathrm{D}}$ is actually the sum of projected velocity dispersions, meaning it is an approximation for the true 3D dispersion. The average dispersion for all stars is $\left\langle\sigma_{3 \mathrm{D}}\right\rangle=179 \pm 5 \mathrm{~km} \mathrm{~s}^{-1}$. For our analysis, we grouped our stars in $r$ bins of 3 arcsec width. This bin size ensures that at least 20 stars are located in each bin. For each bin we computed the observed 3D dispersion according to Eq. (17) and from this (and the number of stars in the bin) a Maxwellian profile. The combined profile for all 664 stars is the superposition of all individual Maxwellians.

Observed and theoretical distributions are compared in Fig. 10. A reduced- $\chi^{2}$ test finds $\chi_{\text {red }}^{2}=1.16$, indicating a good agreement. This tells us that our simple model indeed is within errors - a reasonable approximation of the true speed distribution.

Although there is good agreement between the prediction of this model and the data, it is possible that there are few highvelocity stars which are inconsistent with the global distribution (in terms of our Maxwellian approximation). We therefore examined the number of stars with $v_{3 \mathrm{D}}(r)>2 \sigma_{3 \mathrm{D}}(r)$. From integrating a Maxwellian, we expect this to be the case for a fraction of 0.0074 of the stars in a given ensemble, i.e. this should be the case only for a very small number of stars. In one case, we find 2 out of the 61 stars located in the respective bin showing such high velocities where 0.45 are expected. Using a Monte Carlo test (with 10000 realizations) operating on a Maxwellian distribution for 61 stars, we find the probability for two stars having $v_{3 \mathrm{D}}^{2}(r)>4 \sigma_{3 \mathrm{D}}^{2}(r)$ to be $7.6 \%$ (corresponding to a Gaussian significance of $1.8 \sigma)$ - suggesting this excess is not significant. All stars in our sample are thus compatible with Maxwellian statistics. We therefore conclude that our ensemble of CO stars is consistent we being a uniform, dynamically relaxed system.

This result helps put the high speed of the SiO maser star IRS 9 (cf. Fig. 1) recently discussed by Reid et al. (2007)
Table 1. Properties of the high velocity star S111. Coordinates $x, y$ are given in arcsec, velocities $v$ are given in $\mathrm{km} \mathrm{s}^{-1}$. Errors are statistical.

\begin{tabular}{lcc}
\hline \hline Parameter & Value & Error \\
\hline$x^{a}$ & -1.127 & 0.001 \\
$y^{a}$ & -0.936 & 0.001 \\
$r$ & 1.464 & 0.001 \\
$v_{x}$ & -121 & 5 \\
$v_{y}$ & -308 & 5 \\
$v_{z}$ & -739 & 5 \\
$v_{3 \mathrm{D}}$ & 810 & 9 \\
$v_{<\infty}$ & 788 & $40^{b}$ \\
\hline
\end{tabular}

${ }^{a}$ Position fit for reference epoch 2005.36; ${ }^{b}$ error (statistical + systematic) due to uncertainties of $M_{\bullet}$ and $R_{0}$.

into context. They found a $3 \mathrm{D}$ speed of $\approx 370 \mathrm{~km} \mathrm{~s}^{-1}$ for this star located $0.33 \mathrm{pc}$ away from Sgr A*. They concluded that IRS 9 is too fast to be bound to the mass enclosed within its radial position from Sgr A*. From our analysis (see Fig. 10) one can see that IRS 9 has a high, but not excessive $3 \mathrm{D}$ velocity with respect to the global speed distribution. As we find 11 out of 664 (i.e. $1.7 \%$ ) stars with speeds above $358 \mathrm{~km} \mathrm{~s}^{-1}$ (i.e. $\left.v_{3 \mathrm{D}}^{2}>4\left\langle\sigma_{3 \mathrm{D}}^{2}\right\rangle\right)$, detecting one out of 15 as in the Reid et al. (2007) sample does not appear exceptional.

We however find one more star with a very high speed of $v_{3 \mathrm{D}}=810 \pm 9 \mathrm{~km} \mathrm{~s}^{-1}$. This speed exceeds by far the range of the statistical distribution shown in Fig. 10. Gillessen et al. (2008) identify this star - labeled S111 - as a member of the S-star cluster. Table 1 summarizes the properties of S111.

The projected distance $r$ gives a lower limit for the physical $3 \mathrm{D}$ distance $R$. From this we compute the highest speed possible for a star which is still bound to the black hole:

$v_{<\infty}=\sqrt{\frac{2 G M_{\bullet}}{r}}$.

Hence we see that $\mathrm{S} 111$ might be not bound to $\operatorname{Sgr} A *$. This is in agreement with the findings by Gillessen et al. (2008) who conclude that S111's orbit around Sgr A* might be hyperbolic. It is however possible that $\mathrm{S} 111$ is still bound to the GC star cluster if (a) it follows a highly eccentric orbit and (b) we happen to observe it close to its pericentre. In this case, the stellar mass enclosed by the star's orbit can be (together with Sgr A*) sufficient to bind the star to the cluster. For a more detailed outline of this scenario, see Reid et al. (2007).

S111's high velocity might point towards dynamical interactions different from normal two-body relaxation processes in a near-thermal stellar population. In a recent analysis, Perets et al. (2007) point out that the presence of so-called massive perturbers (mainly giant molecular clouds) in the $\mathrm{GC}$ region might lead to a substantial number of close encounters between binary stars and Sgr A*. Such three-body interactions can result in binary disruption with one of the stars being ejected from the GC with a speed up to several thousand $\mathrm{km} \mathrm{s}^{-1}$ (Hills 1988).

This scenario is of interest especially in view of Galactic hypervelocity stars (HVS) which have speeds higher than the escape speed of the Milky Way (although this is not the case for S111 itself). Right now, 16 of these stars with GC distances in the range $\approx 30 \ldots 130 \mathrm{kpc}$ are known (Brown et al. 2005, 2007; Edelmann et al. 2005; Hirsch et al. 2005; Brown et al. 2008). These stars are assumed to have been ejected from the Galactic centre according to the mechanism proposed by Hills (1988), although Przybilla et al. (2008) and Bonanos et al. (2008) recently 

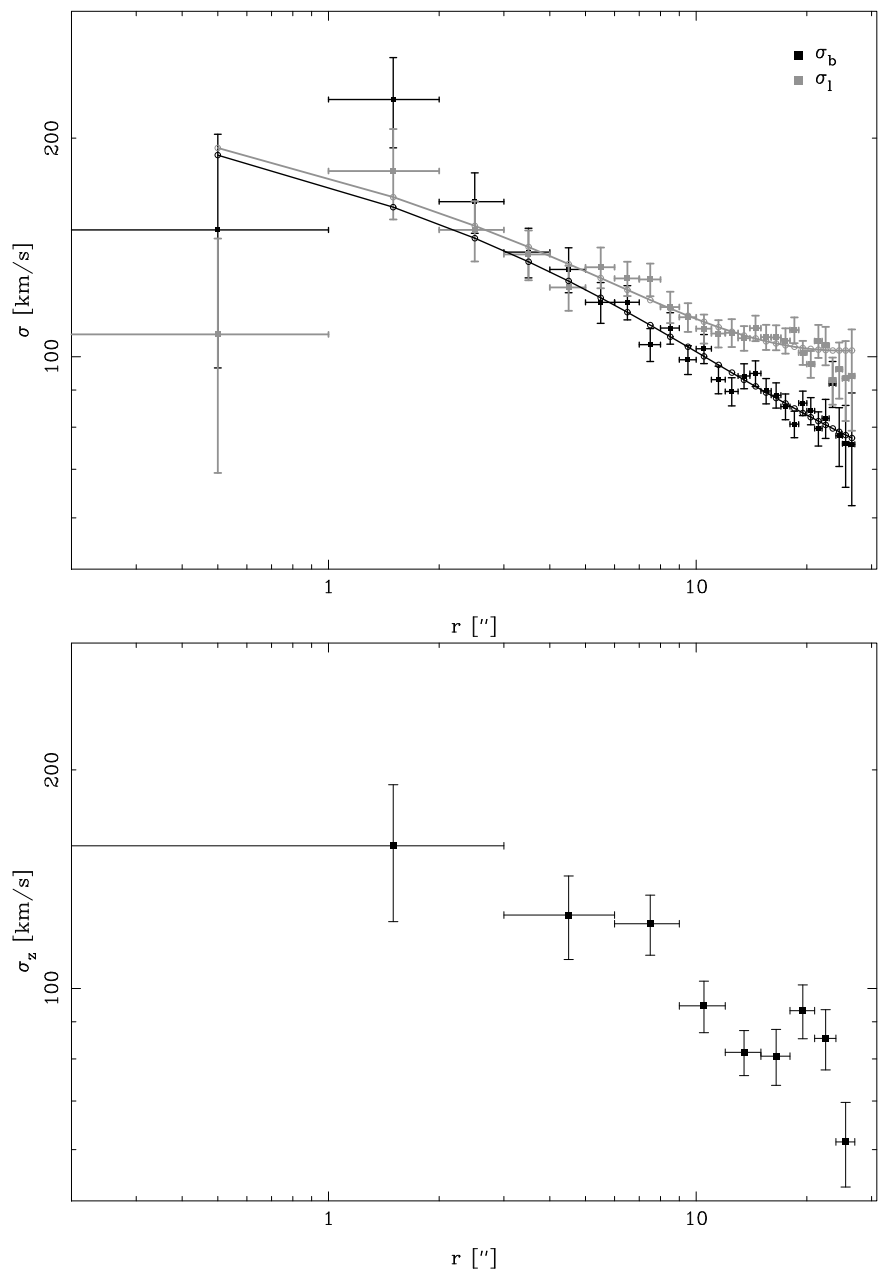

Fig. 11. Velocity dispersion profiles. Top panel: proper motion dispersions. Squares with error bars are observed velocity dispersions $\sigma_{l, b}$ vs. projected distance from $\mathrm{Sgr}^{*} * r$. Continuous lines mark the best-fitting cluster model assuming a spherical, rotating system (please see the text for details). Bottom panel: observed radial velocity dispersions. As the number of 3D velocity stars is about nine times smaller than the number of $2 \mathrm{D}$ velocity stars, the profile is sampled sparsely compared to the proper motion dispersion profile.

concluded that one of these stars actually originates from the Large Magellanic Cloud.

In summary, we can conclude the following: (1) the distribution of the stellar 3D speeds confirms the locally relaxed nature of the GC late type cluster. (2) We might have found one highvelocity star unbound to Sgr A*.

\subsection{Kinematic modelling}

The large number of proper motions we have at hand allow us to compute a densely sampled velocity dispersion profile. We calculated the velocity dispersions $\sigma_{l, b}$ as functions of projected distance from Sgr A* $r$ for $r<27$ ', The resulting distributions are shown in Fig. 11 (along with the corresponding profile for $\left.\sigma_{z}\right)$. Within the errors, both profiles $\left(\sigma_{l, b}\right)$ decrease monotonically with increasing projected distance. With increasing $r$, the two profiles diverge due to rotation and possibly anisotropy.

Additional information is provided by the rotation profile $\left\langle v_{z}\right\rangle(l)$ obtained from radial velocity data. For $|l| \leq 24^{\prime \prime}$ we make use of our SINFONI results (see Fig. 8, bottom panel). We also

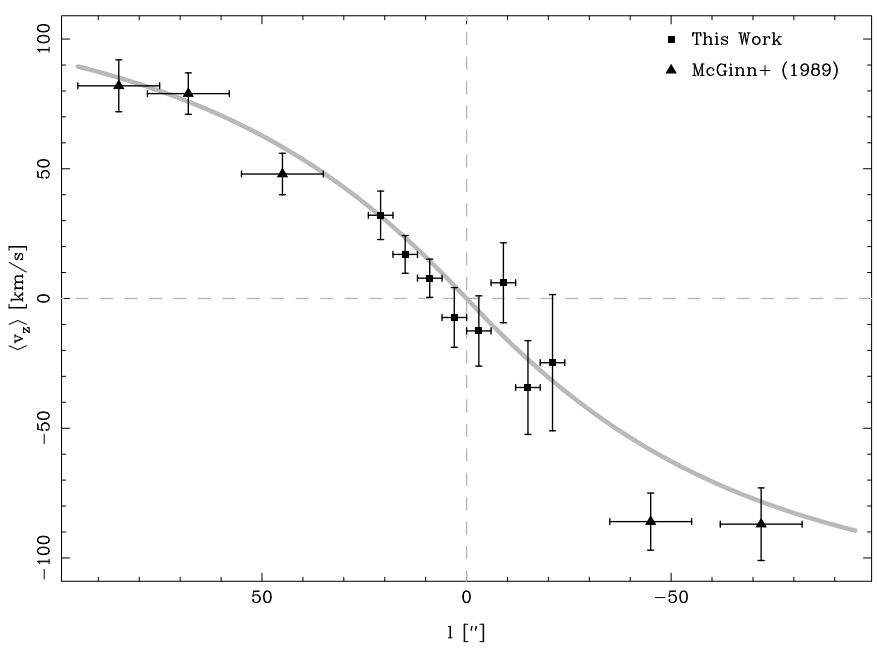

Fig. 12. Average radial velocity vs. Galactic longitude $l$ as derived from our modelling (continuous grey line). For comparison, the observed values (black data points with error bars) are given. The data are from our work and from McGinn et al. (1989).

included data from McGinn et al. (1989) covering the range $|l|=45 \ldots 85^{\prime \prime}$; see Fig. 12 for an overview. In the following discussion we neglect the fact that $\left\langle v_{z}\right\rangle(l)$ was not measured exactly at $b=0$ but in a strip $|b| \lesssim 10^{\prime \prime}$; we found that this has no significant impact.

In order to quantify the cluster's dynamical properties we simultaneously analysed the proper motion dispersion and rotation profiles. We used an edge-on rotating, spherical stellar system as a model. We fit the model parameters to the data by means of a $\chi^{2}$ minimization. Coordinates $l, b, z$ are Galactic longitude, Galactic latitude, and l.o.s. axis respectively. Our model contains spherical coordinates $R, \theta, \phi$; these are the 3D distance from Sgr $\mathrm{A}^{*}$, the zenith angle (with $\theta=0(\pi)$ being (anti)parallel to the $b$ axis, $\theta=\pi / 2$ being parallel to the $l$ axis), and the azimuth angle respectively. The projected $2 \mathrm{D}$ distance from $\mathrm{Sgr} \mathrm{A}^{*}$ is denoted by $r$.

For our model fit we solve the equations

$$
\begin{aligned}
& \Sigma(r) \sigma_{b}^{2}(r)=2 \int_{r}^{\infty} \sigma_{b}^{2}(R) \frac{\rho(R) R}{\sqrt{R^{2}-r^{2}}} \mathrm{~d} R \\
& \Sigma(r) \sigma_{l}^{2}(r)=2 \int_{r}^{\infty}\left[\sigma_{l}^{2}(R)+\tilde{v}_{\phi}(R)^{2}\left(1-\frac{r^{2}}{R^{2}}\right)\right] \frac{\rho(R) R}{\sqrt{R^{2}-r^{2}}} \mathrm{~d} R \\
& \Sigma(r=|l|) v_{z}(|l|)=2 \int_{r=|l|}^{\infty} v_{\phi}(R, \theta=\pi / 2) \frac{r}{R} \frac{\rho(R) R}{\sqrt{R^{2}-r^{2}}} \mathrm{~d} R
\end{aligned}
$$

with

$\Sigma(r)=2 \int_{r}^{\infty} \rho(R) \frac{R}{\sqrt{R^{2}-r^{2}}} \mathrm{~d} R$.

$\Sigma(r)$ is the cluster's stellar surface mass density in the sky plane (not the one of the sample population; see the discussion in Sect. 3.1), $\rho(R)$ is the $3 \mathrm{D}$ stellar volume mass density, $\sigma_{l, b}(R)$ is the intrinsic velocity dispersion, and $v_{\phi}(R, \theta)$ is the rotation speed (e.g. Wilson 1975; Binney \& Tremaine 1987; Gerhard 1994; Genzel et al. 1996, 2000). 
In the integrations we use the parametrized functions

$$
\begin{aligned}
& v_{\phi}(R, \theta)=v_{r} \frac{R \sin \theta}{\sqrt{R^{2} \sin ^{2} \theta+\eta^{2}}} \\
& \tilde{v}_{\phi}(R)=\frac{1}{\pi} \int_{0}^{\pi} v(R, \theta) \mathrm{d} \theta \\
& \rho(R)=\frac{\rho_{0}}{1+\left(R / R_{b}\right)^{2}} \\
& \sigma_{b}^{2}(R)=\sigma_{0}^{2}\left(R / R_{z}\right)^{2 \alpha}+\sigma_{\infty, b}^{2} \\
& \sigma_{l}^{2}(R)=\sigma_{0}^{2}\left(R / R_{z}\right)^{2 \alpha}+\sigma_{\infty, l}^{2} .
\end{aligned}
$$

In this parametrization, $\rho(R)$ is given as a powerlaw sphere profile with break radius $R_{b} ; \sigma_{l, b}(R)$ is given as a superposition of a powerlaw dispersion profile and a - possibly anisotropic - constant floor dispersion at infinite distances $\sigma_{\infty, l, b}$. The rotation velocity is described by a profile rising from 0 to an asymptotic speed $v_{r}$ with a characteristic scale $\eta$.

We selected the parametrization of the density profile according to the results from observations of stellar number counts and $K$-band surface brightness distributions ${ }^{5}$ (Launhardt et al. 2002; Genzel et al. 2003; Mouawad et al. 2005; Schödel et al. 2007). However, the characteristic scale $\eta$ is not well constrained by the observations; reported values range from $\approx 6^{\prime \prime}$ (Launhardt et al. 2002) to $\approx 10^{\prime \prime}$ (Genzel et al. 2003). Therefore we introduce $\eta$ as a fit parameter into our model because it is not a priori clear that the $\eta$ which fits best the kinematics is the same as the density profile break radius.

Since for our purposes the normalization factors $\rho_{0}, R_{z}$ (Eqs. (25)-(27)) are arbitrary, we fixed them to 1 . It is wellknown that the cluster is dominated by the point mass Sgr A*; we therefore selected a Keplerian profile for the velocity dispersion, i.e. $\alpha=-0.5$.

In summary, we fit for the 6 fit parameters $\left\{\sigma_{0}, \sigma_{\infty, b}, \sigma_{\infty, l}\right.$, $\left.R_{b}, v_{r}, \eta\right\}$. We used the Mathematica FindMinimum ${ }^{6}$ routine to fit the model to the observed dispersion profiles $\sigma_{l, b}(r)$. From the best-fitting model we found

$$
\begin{aligned}
& \sigma_{0}=359 \pm 30 \mathrm{~km} \mathrm{~s}^{-1} \\
& \sigma_{\infty, b}=54.2 \pm 8.0 \mathrm{~km} \mathrm{~s}^{-1} \\
& \sigma_{\infty, l}=55.4 \pm 8.8 \mathrm{~km} \mathrm{~s}^{-1} \\
& R_{b}=8.9 \pm 3.5 \operatorname{arcsec} \\
& v_{r}=189 \pm 38 \mathrm{~km} \mathrm{~s}^{-1} \\
& \eta=109 \pm 44 \operatorname{arcsec}
\end{aligned}
$$

Parameter errors are statistical $\left(68 \%\right.$ confidence level, $\Delta \chi^{2}=$ 7.17). The fact that some of these errors are relatively large mirrors correlations between parameters. We find $\chi_{\text {red }}^{2} \equiv \chi^{2} / F=$ $0.79 ; F=61$ is the number of degrees of freedom. This shows a good agreement between model and observational data. The resulting model profiles are presented in Figs. 11, 12 together with the corresponding data.

\footnotetext{
5 In this discussion, we assume a constant mass-to-light ratio.

6 Wolfram Research, Inc., Champaign, IL, USA.
}

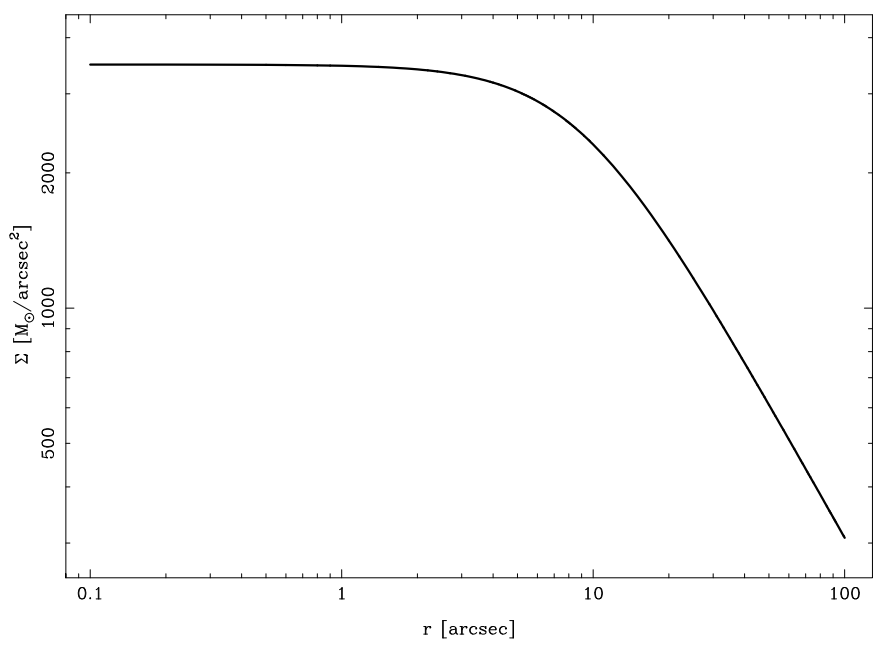

Fig. 13. Stellar surface mass density $\Sigma$ vs. projected distance $r$ from Sgr A* for the model cluster.

We can now draw several conclusions. The main assumptions our model is based on, especially stellar density profile and velocity dispersion profiles, actually allow for a reasonable fit $\left(\chi_{\text {red }}^{2} \lesssim 1\right)$. We can thus be confident that our model description is sufficiently complete. Our result $R_{b} \approx 9^{\prime \prime}$ is in good agreement with the observations of the density distribution. Figure 13 shows the stellar surface density as found by our modelling. The different scalings (mass densities vs. observed number densities) aside, this profile is in good agreement with the earlier observations discussed above.

As $\sigma_{\infty, l}=\sigma_{\infty, b}$ within the errors, the nuclear cluster can be described as an isotropic system.

Until now, our discussion was focused on the kinematic description of the nuclear cluster. In the following, we will draw dynamical conclusions.

Since we have analytic parametrizations for the stellar density profile (Eq. (25)), dispersion profile (Eqs. (26), (27)), and rotation profile (Eqs. (23), (24)) at hand, we can compute the mass profile of the cluster. For a spherical, isotropic, rotating system, the mass distribution is given by the Jeans equation via

$G M(R)=-R \sigma^{2}\left(\frac{\mathrm{d} \log \rho}{\mathrm{d} \log R}+\frac{\mathrm{d} \log \sigma^{2}}{\mathrm{~d} \log R}-\frac{v_{\phi}^{2}}{\sigma^{2}}\right)_{R}$

(e.g. Binney \& Tremaine 1987). This relation allows us to compute the enclosed mass as a function of $R$. A non-zero value at $R=0$ should correspond to a central point mass, in our case the mass of Sgr A*.

In Fig. 14 we show the results found when inserting our bestfitting model parameters (black curves). Contrary to the known value $M_{\bullet} \simeq 4 \times 10^{6} M_{\odot}$ (Schödel et al. 2002, 2003; Ghez et al. 2003, 2005, 2008; Eisenhauer et al. 2005; Gillessen et al. 2008), the total enclosed mass drops down to $M(R \rightarrow 0) \simeq 1.2 \times$ $10^{6} M_{\odot}$. The formal statistical $1 \sigma$ uncertainty of this value is $\approx 15 \%$, meaning the discrepancy is systematic.

This inconsistency in the behaviour of Jeans equation mass profiles in star cluster cores was already noted by Kormendy \& Richstone (1995). They conclude that a good sensitivity to central point masses is given only in the case of steep $(\mathrm{d} \log \rho / \mathrm{d} \log R \lesssim-2)$ density profiles. If the central core of a stellar system is resolved (leading to $\mathrm{d} \log \rho / \mathrm{d} \log R \approx 0$ for $R \rightarrow 0$ ), the enclosed mass drops systematically compared to measurements using the large-scale, "intrinsic" slope of the 


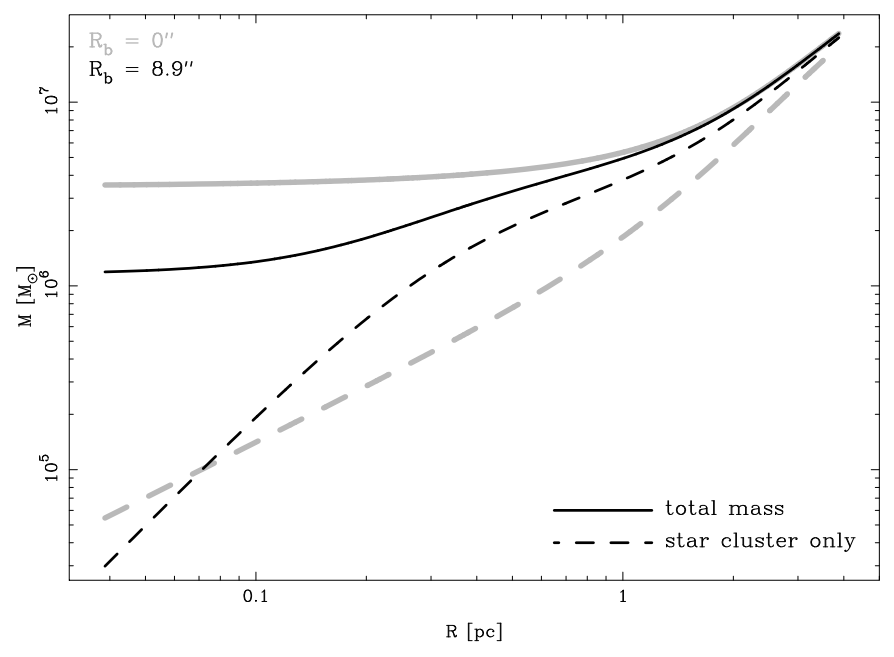

Fig. 14. Enclosed mass vs. distance from $\mathrm{Sgr} \mathrm{A}^{*}$ calculated via the Jeans equation for $1^{\prime \prime}<R<100^{\prime \prime}$. The continuous black curve is the mass profile using our best-fitting model density profile with break radius $R_{b}=8.9^{\prime \prime}$. The continuous grey line shows the profile obtained when neglecting the flattening of the density profile towards small $R$, i.e. assuming $R_{b}=0$. The corresponding dashed curves are the stellar mass profiles obtained by subtracting the central point masses (i.e. the values found at $R=0$ ). The scale is $1 \mathrm{pc} \equiv 25.8^{\prime \prime}$.

density profile. This behaviour is obvious from Eq. (28). We therefore might interpret our findings as due to a "resolution effect". This is especially interesting in view of the fact that the central few arcseconds of the nuclear cluster are dominated by a different stellar population. Whereas we analyse the properties of CO late-type stars, towards small $R$ an increasing fraction of the stars belongs to a population of young early-type stars. Therefore the intrinsic slope of the density profile in the central part of the cluster is not obvious at all.

In order to check the influence of this "resolution effect", we repeated our calculations neglecting the flattening of the density profile towards the cluster centre, i.e. assuming $R_{b}=0$. The resulting mass profiles are shown in Fig. 14 (grey curves). Here we indeed find $M(R \rightarrow 0) \simeq 3.6 \times 10^{6} M_{\odot}$, in agreement with the mass of Sgr A* (within the errors). For $R>1 \mathrm{pc}$, the discrepancy between the profiles vanishes. However, we do not conclude from this that we have found the true mass of the central SMBH by applying the proper assumption. Instead, the range of results should be read as the systematic uncertainty intrinsic to this type of mass estimates. This means that the Jeans profile ansatz allows us to derive the correct order of magnitude (a few $10^{6} M_{\odot}$ in our case) of the central point mass, but with a factor $\approx 2$ systematic uncertainty.

In any case $\left(R_{b}=8.9^{\prime \prime}\right.$ or $\left.R_{b}=0\right)$, we can derive the enclosed stellar masses $M_{*}(R)$ by subtracting the corresponding central point masses. The resulting profiles are given in Fig. 14 (black and grey dashed curves). Within the systematic uncertainties, the profiles begin at $M_{*}\left(R=1^{\prime \prime}\right) \simeq 4 \times 10^{4} M_{\odot}$ and rise to $M_{*}\left(R=100^{\prime \prime}\right) \simeq 2.3 \times 10^{7} M_{\odot}$. Although this is just consistent with earlier studies (e.g. Lindqvist et al. 1992; Ghez et al. 1998; Genzel et al. 1996, 2000, and references therein), our masses are systematically higher by factors $\approx 1.5$. This is due to the fact that we find a faster rotation of the cluster than the aforementioned works. For the same reason, we find a somewhat smaller sphere of influence (meaning the radius where $M_{*}(R)=M_{\bullet}$; Alexander 2005 ) for $\operatorname{Sgr} \mathrm{A}^{*}$ which is $\approx 1.5 \mathrm{pc}$ (instead of $\approx 3 \mathrm{pc}$; Alexander 2005).
In order to calibrate the $\Sigma$-axis of Fig. 13, we adopted the following approach. We use Eq. (28) to compute the enclosed mass $M(R)$ at a reference distance $R$ which is large enough to avoid the range where the mass profile shows substantial uncertainties but still within the range covered by data (see Fig. 12). We picked $R=47$ '. For this $R$, the expressions given in the $(\cdots)$ part of Eq. $(28)$ are $\approx-1.9, \approx-0.5$, and $\approx 1.0$, respectively. From this we find $M\left(R=47^{\prime \prime}\right) \simeq 8.3 \times 10^{6} M_{\odot}$. Subtracting the contribution by $\operatorname{Sgr} A^{*}\left(M_{\bullet} \simeq 4 \times 10^{6} M_{\odot}\right)$ leaves us with an enclosed stellar mass $M_{*}\left(R=47^{\prime \prime}\right) \simeq 4.3 \times 10^{6} M_{\odot}$. With this value, we obtain $\rho_{0} \simeq 2.1 \times 10^{6} M_{\odot} / \mathrm{pc}^{3}$ (see Eq. (25)).

\subsection{Phase-space distributions}

As shown in Sects. 5.1-5.3, the observed part of the GC star cluster can approximately be described as a spherical rotator with normally distributed random stellar velocities. It is, however, not clear if the observed stars kinematically indeed form a single system. A well-known example for kinematic segregation in the GC is the dichotomy between isotropically distributed old late-type stars and young early-type stars arranged in disks (Genzel et al. 2003; Paumard et al. 2006).

A common way to characterize a stellar system is the use of phase-space maps (e.g. Ibata et al. 2001; Yanny et al. 2003; Martinez-Delgado et al. 2004; Seabroke \& Gilmore 2007). Applying this method to our data, we construct a variety of phase-space diagrams. The resulting distributions are presented in Figs. 15 and 16. Qualitatively, there appears to be no substructure or grouping. In the $v_{z}$-l-diagram the data are biased towards positive $l$. This is an observational artefact as SINFONI spectra were preferentially obtained north of $\operatorname{Sgr} \mathrm{A}^{* 7}$, roughly corresponding to $l>0$.

The phase-space maps also mirror the influence of global rotation and anisotropy discussed in the previous subsections. These effects show up as a broadening of the $v_{l}$-vs.-coordinate distributions along the velocity axes compared to the respective distributions for $v_{b}$. However, one can hardly recognize the rotation pattern from the $v_{z}-l$ plot as the random scatter of the data points (i.e. the dispersion) is much larger than the modulation in the velocity average (cf. Fig. 8, bottom panel).

In order to quantify the presence (or absence) of phase-space substructure, we made use of the two-point correlation function (TPCF)

$\xi(s)=\frac{n_{R}}{n_{D}} \frac{D D(s)}{D R(s)}-1$

(Davis \& Peebles 1983). For a given distance $s, D D(s)$ is the number of pairwise distances between observed stars (also referred to as the data-data distances) located in the corresponding distance bin. $D R(s)$ is the number of pairwise distances between the observed stars and the members of a comparison ensemble, usually a random, uniform one (thus $D R(s)$ is also referred to as the data-random distances). $n_{D}\left(n_{R}\right)$ is the total number of datadata (data-random) distances. By definition, $\xi(s)$ is located in the range $[-1 ;+\infty] ; \xi(s)=0$ corresponds to full agreement between observed ensemble and comparison ensemble.

We computed the TPCF for all 5445 proper motion stars. Since, in phase-space, the distance parameter $s$ mixes positions and velocities, we used a normalized 4D distance

$$
s=\sqrt{\left(\Delta l / x_{0}\right)^{2}+\left(\Delta b / x_{0}\right)^{2}+\left(\Delta v_{l} / v_{0}\right)^{2}+\left(\Delta v_{b} / v_{0}\right)^{2}}
$$

7 Because SINFONI's AO guide star is located $\approx 17$ " north of Sgr A*. 

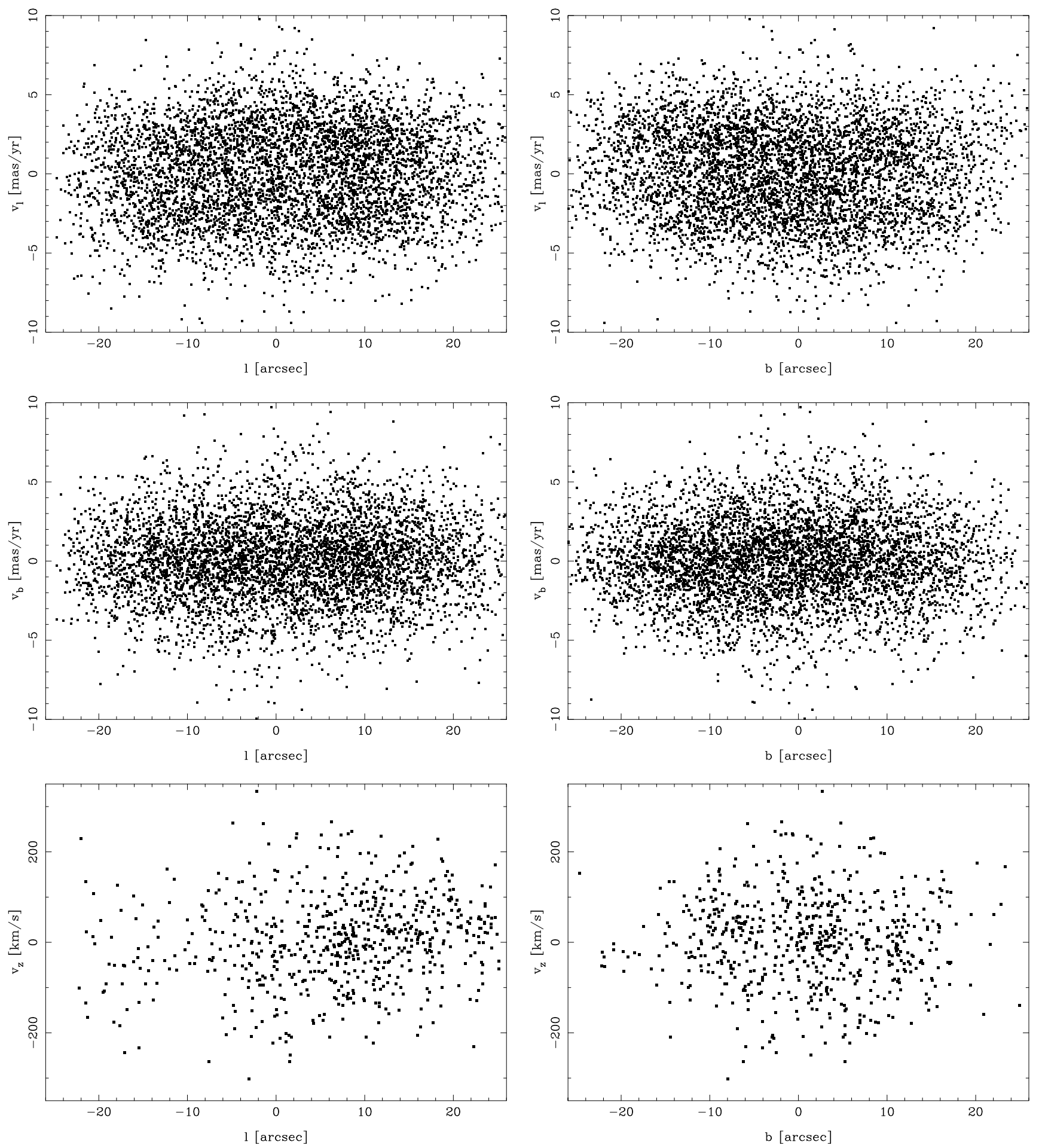

Fig. 15. Velocity-coordinate phase-space maps. Velocities in the sky plane $v_{l, b}$ include all 5445 proper motion stars; line-of-sight velocities $v_{z}$ are given for 664 stars. The scale is $1 \mathrm{mas} / \mathrm{yr} \equiv 37.9 \mathrm{~km} \mathrm{~s}^{-1}$. Compared to the $v_{b}$, the $v_{l}$ distributions are broadened (in velocity) due to the global rotation. The $v_{z}$ are biased towards positive $l$ as SINFONI spectra were collected mainly north of Sgr A*. These global properties aside, the diagrams show no obvious patterns or sub-structures.

Here $x_{0}, v_{0}$ are (a priori arbitrary) constant distances and velocities; $\Delta$ denotes the difference in the given coordinate. In order to match the phase-space dimensions of the cluster (cf. Figs. 15, 16), we chose $x_{0}=1 \mathrm{pc}=25.8^{\prime \prime}, v_{0}=$ $400 \mathrm{~km} \mathrm{~s}^{-1}=10.55 \mathrm{mas} / \mathrm{yr}$ (with $R_{0}=8 \mathrm{kpc}$ ). Thus $\mathrm{s}=1$ corresponds to the half side length of a "phase space unit cell".

As a first step, we computed the TPCF using a random, uniformly distributed comparison ensemble. The resulting distribution is shown in Fig. 17 (left hand panel). It mirrors the obvious clustering of phase-space points around $\left(l, b, v_{l}, v_{b}\right)=$ $(0,0,0,0)$ shown in Figs. 15,16 . The point where the profile crosses the $\xi(s)=0$ line can be identified as a characteristic phase-space radius of the cluster; this radius is $s_{\mathrm{c}} \simeq 1.05$.

As a second step, we computed the TPCF using a Monte Carlo model of the cluster. This model is a random realization of the kinematic model solution described in the previous 

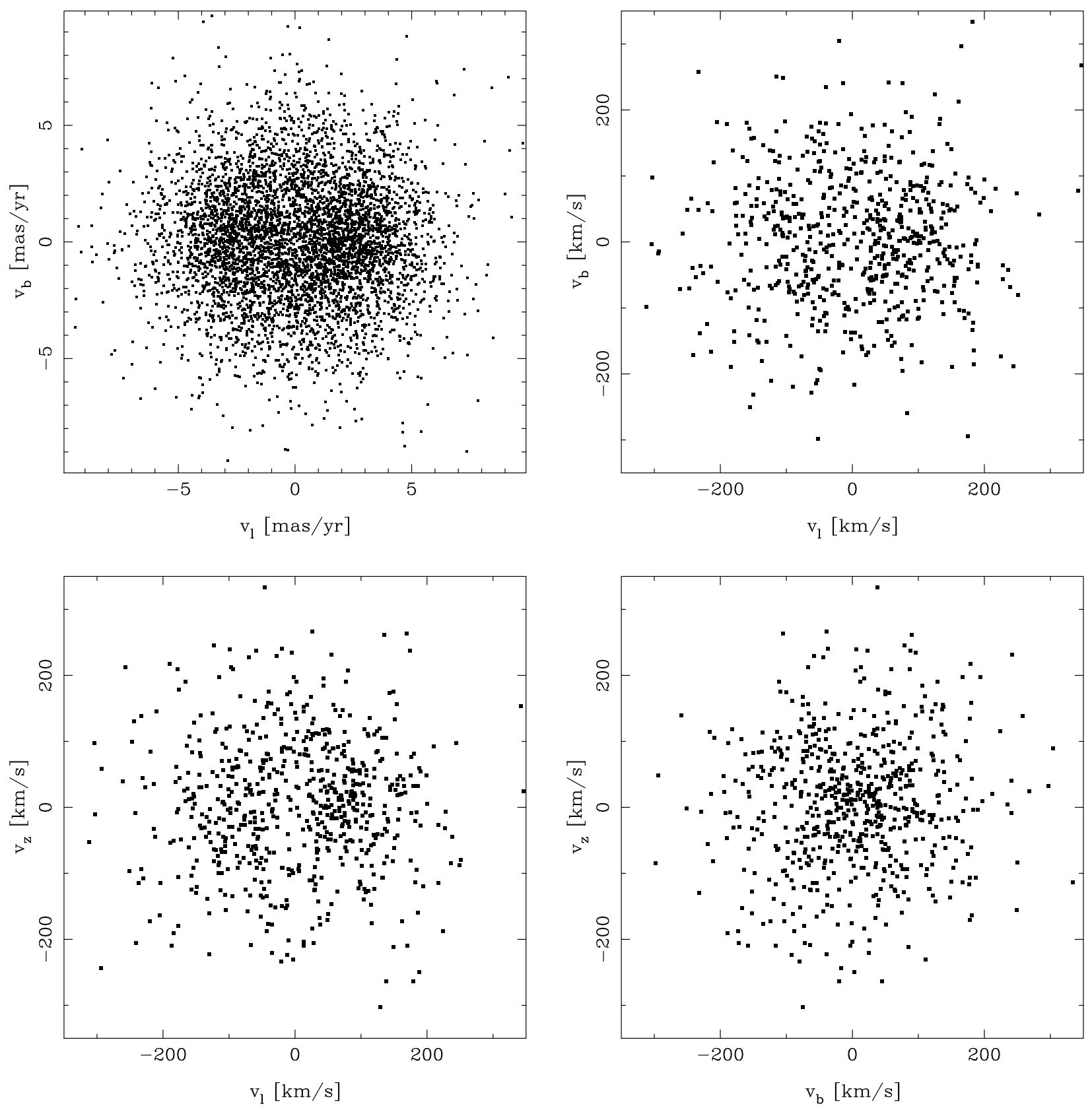

Fig. 16. Velocity-velocity phase-space maps. The distribution of $v_{l}$ vs. $v_{b}$ is given twice, once for all 5445 proper motion stars (top left) in mas/yr, once for all $6643 \mathrm{D}$ motion stars (top right) in $\mathrm{km} \mathrm{s}^{-1}$. The scale is $1 \mathrm{mas} / \mathrm{yr} \equiv 37.9 \mathrm{~km} \mathrm{~s}^{-1}$. In analogy to Fig. 15, the global rotation shows up as a broadening of the $v_{l, z}$ distributions with respect to $v_{b}$ (see also Figs. 9, 18). These global properties aside, there is no obvious substructure or grouping.

section. We extracted model data by applying an "on-sky" selection mask simulating the actual imaging observations and the selection of stars (FOV, minimum star-star distances). These values were inserted into the TPCF calculation.

The right hand panel of Fig. 17 shows the resulting $\xi(s)$ profile. In general, data and model are in very good agreement, deviations are typically of the order $<1 \%$ (from $\xi(s)+1=1$ ). The largest deviations from data-model equality, about $4 \%$, occur at $s \approx 0.15$. Such a signal at small $s$ corresponds to a slight excess of stars with small pairwise phase-space distances compared to the model distribution. This would mean that either (1) the model distribution slightly underestimates the number of stars with small $s$ or (2) the cluster contains a small excess (with respect to a random sample) population of stars moving coherently. However, in either case it is safe to conclude that our - quite simple - kinematic model indeed reproduces the observed projected phase-space distribution of the GC cluster.

We did not include stellar radial velocities in the TPCF analysis. The spectra were extracted from 24 separate SINFONI data sets with different FOVs, pointings, pixel scales, integration times, spectral ranges, PSFs, and limiting magnitudes (see Sect. 4.1). This prevented a consistent reconstruction of the observation/selection mask, thus excluding the reliable extraction of a model cluster.

In total, we can conclude that the GC star cluster is a uniform, well phase-mixed system. This is in good agreement with the age estimate for the cluster. Maness et al. (2007) find constant star formation for $>12$ Gyr, meaning that most stars 

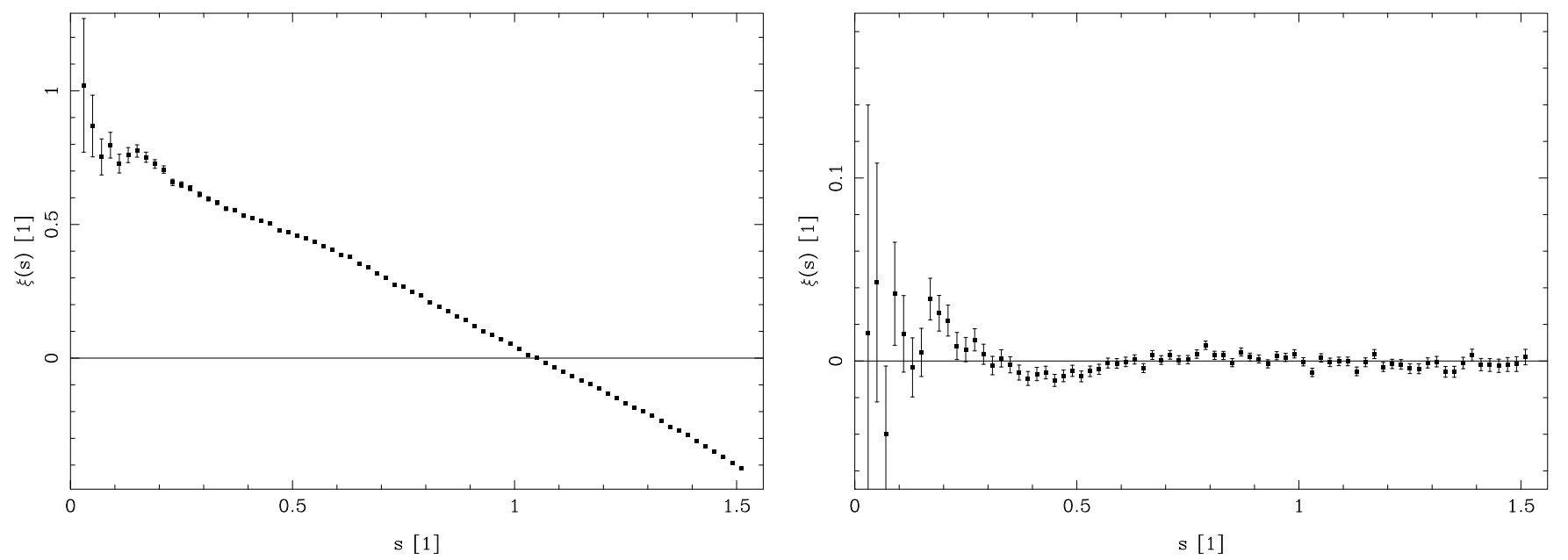

Fig. 17. 4D two-point correlation functions for all proper motion stars. The normalized distance $s$ is defined via $s^{2}=\left(\Delta l / x_{0}\right)^{2}+\left(\Delta b / x_{0}\right)^{2}+$ $\left(\Delta v_{l} / v_{0}\right)^{2}+\left(\Delta v_{b} / v_{0}\right)^{2} ; x_{0}, v_{0}$ are constants. Please note the different $\xi(s)$ axis scales. Left hand panel: measured phase-space distances compared to a uniform random distribution. The profile mirrors the obvious clustering of phase-space points around $\left(l, b, v_{l}, v_{b}\right)=(0,0,0,0)$ shown in Figs. 15,16 . Right hand panel: measured phase-space distances compared to a Monte-Carlo model of the GC cluster. Deviations from the uniform rotator model cluster do not exceed $\approx 0.04$.

are older than $\approx 10^{9} \mathrm{yr}$, the cluster's two-body relaxation time (Alexander 2005). In the following, we will discuss how our findings might help to constrain the dynamical history of the nuclear cluster. The most important question here is: if the GC cluster experienced the infall of another stellar system (e.g., a small star cluster), would we be able to detect corresponding kinematic traces in our data?

This discussion ties in with the debate on the origin of the young ( $\approx 6 \mathrm{Myr}$ ) early-type stars located in two disks centered on Sgr $A^{*}$, for which two mechanisms have been proposed: in situ star formation (e.g. Levin \& Beloborodov 2003; Goodman 2003) or infall of a star cluster (e.g. Gerhard 2001; McMillan \& Portegies Zwart 2003). In their analysis, Paumard et al. (2006) conclude that the initial mass of an infalling cluster is limited to $\approx 17000 M_{\odot}$ for the more massive disk. In contrast, the inpiraling-cluster-scenario requires initial cluster masses $>10^{5} M_{\odot}$. From this, Paumard et al. (2006) conclude that the inpiraling-cluster-scenario is highly unlikely.

On the one hand, the limited number of data points (in phase space) sets a lower limit on the numbers of stars involved in or affected by such an event. Events involving only a small fraction of the stellar population would be masked by Poisson noise and residual methodological uncertainties. For somewhat larger events, the cluster returns to a relaxed, (quasi) equilibrium state within about one two-body relaxation time; this time is about 1 Gyr (Alexander 2005). However, phase-mixing (e.g. Binney \& Tremaine 1987) might erase the phase-space signature of any infall event already within few dynamical times $\left(t_{\text {dyn }} \approx 10^{5}\right.$ yr for the GC cluster; Alexander 2005)

On the other hand, violent relaxation (Lynden-Bell 1967; for a recent review, see Bindoni \& Secco 2008, and references therein) sets an upper limit on the amount of a dynamical distortion we could detect. In case of the infall of a massive object (in the order of the mass of the nuclear cluster), the cluster returns to a relaxed, (quasi) equilibrium state within about one dynamical time. This means that we would miss infalls of massive objects longer ago than few $10^{5}$ years anyway.

To summarize this discussion: we do not detect obvious kinematic signatures of an infall event. This indicates that there has been no major distortion of the GC cluster at least within the last few $10^{5}$ years. At this stage we are not able to quantify our statement further. We do expect, however, that a more detailed analysis, possibly in combination with dynamical modelling, will make the phase-space distribution analysis a valuable tool for characterising the kinematics of the GC cluster.

\subsection{Statistical parallax of the Galactic centre}

The availability of 3-dimensional velocity vectors for several hundred stars allows the computation of the distance to the Galactic centre $R_{0}$ using the statistical parallax. If the stellar velocities are distributed isotropically, then the three velocity dispersions $\sigma_{x}, \sigma_{y}, \sigma_{z}$ are equal. As $\sigma_{x}, \sigma_{y}$ are measured in angular units (mas/yr) whereas $\sigma_{z}$ is measured in physical units $\left(\mathrm{km} \mathrm{s}^{-1}\right)$, the distance scale can be derived directly.

As discussed in the previous sections, in case of the GC cluster the global isotropy is broken by rotation. For the three velocity dispersions in $l, b$, and $z$ of our $6643 \mathrm{D}$ velocity stars we find the values

$\sigma_{l}=2.928 \pm 0.082 \mathrm{mas} / \mathrm{yr}$

$\sigma_{b}=2.531 \pm 0.071 \mathrm{mas} / \mathrm{yr}$

$\sigma_{z}=102.3 \pm 2.8 \mathrm{~km} \mathrm{~s}^{-1}$.

The respective distributions are shown in Fig. 18. Our value for $\sigma_{z}$ is in excellent agreement with the value of $100.9 \pm 7.7 \mathrm{~km} \mathrm{~s}^{-1}$ found by Figer et al. (2003) who analysed a smaller sample of 85 CO absorption line stars (recently, Zhu et al. 2008, confirmed this result).

In order to calculate a consistent distance estimate, we need to take into account two effects:

(1) The cluster is rotating in the $b=0$ plane. We therefore use the assumption that the nuclear cluster is an axisymmetric elliptical system with respect to the $b$ axis. This means that $\sigma_{l}$ and $\sigma_{z}$ (but not $\sigma_{b}$ and $\sigma_{z}$ ) can be compared in order to calculate the statistical parallax. 

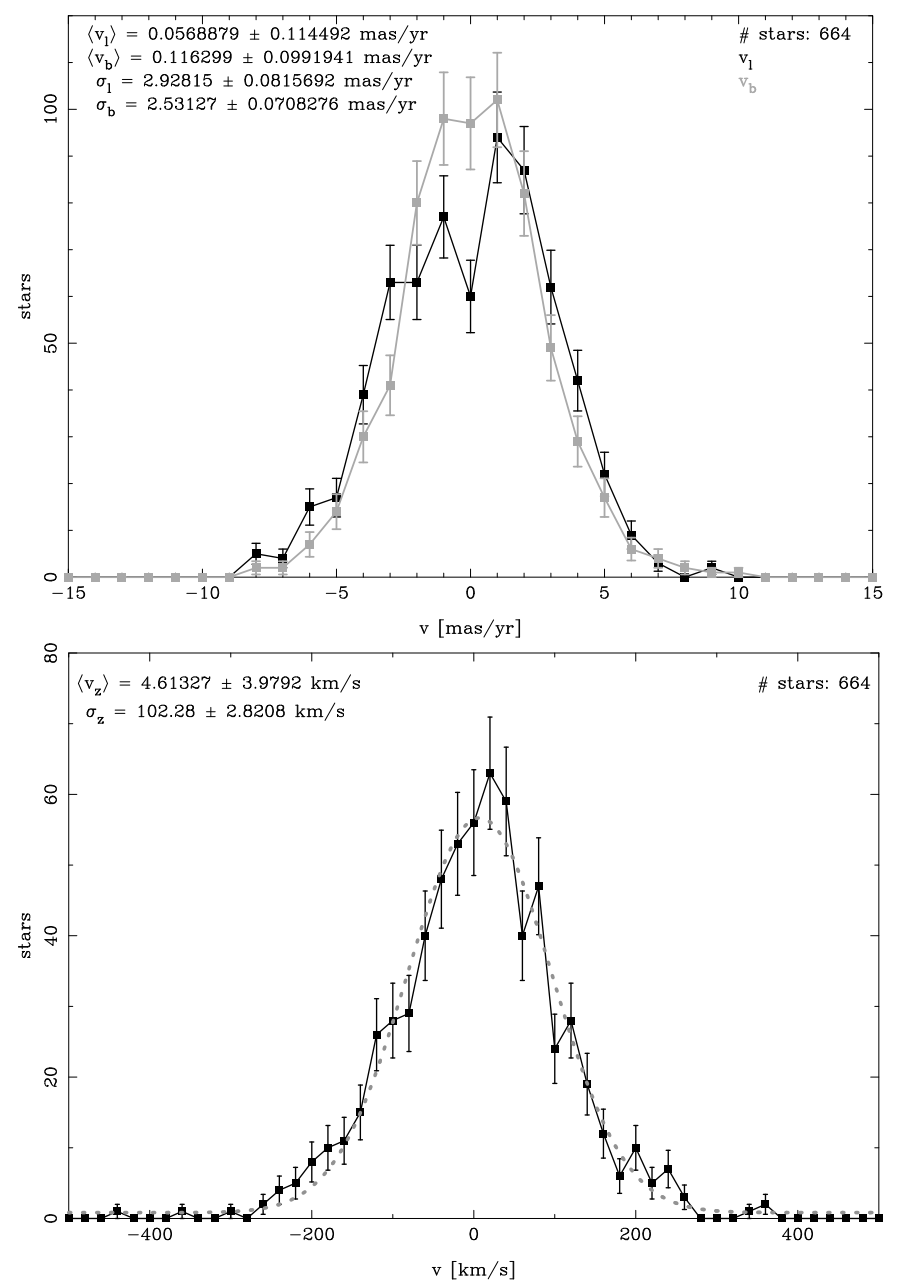

Fig. 18. Histograms of proper motions in $l$ and $b$ (top panel) and of radial velocities (bottom panel) including all stars with measured 3D velocities. The dotted curve in the bottom panel corresponds to the bestfitting Gaussian profile. Error bars mark the Poisson errors. In both plots the respective average velocities $\left\langle v_{l, b, z}\right\rangle$ and velocity dispersions $\sigma_{l, b, z}$ are given.

(2) We need to include projection effects which affect the impact of rotation. Whereas the observed $\sigma_{l}$ is given by Eq. (20), the observed $\sigma_{z}$ is given by the relation

$$
\Sigma(r) \sigma_{z}^{2}(r)=2 \int_{r}^{\infty}\left(\sigma_{l}^{2}(R)+\tilde{v}(R)^{2} \frac{r^{2}}{R^{2}}\right) \frac{\rho(R) R}{\sqrt{R^{2}-r^{2}}} \mathrm{~d} R .
$$

This relation differs from Eq. (20) in the geometry factor multiplied with $\tilde{v}^{2}(R)$; especially, this factor implies that $\sigma_{l}>\sigma_{z}$ in projection for a finite FOV. The use of $\sigma_{l}(R)$ to calculate $\sigma_{z}(r)$ is due to the assumption of axisymmetry.

From a comparison of Eqs. (20) and (31) we obtain a projection correction factor

$k=\frac{\sigma_{l}(r \rightarrow \mathrm{FOV})}{\sigma_{z}(r \rightarrow \mathrm{FOV})} \geq 1$.

Here $\sigma_{l, z}(r \rightarrow$ FOV $)$ are the projected velocity dispersions of the entire sample calculated in identical units (either mas/yr or $\mathrm{km} \mathrm{s}^{-1}$ ). The case $k=1$ corresponds to an infinite FOV; for FOVs covering only the central part of the cluster, $k>1$. From this relation we obtain the effective velocity dispersion in $z$,

$\sigma_{z}^{\prime}=k \sigma_{z}=\sigma_{l}$ where the second equality is given by the assumption of axisymmetry. From our calculations we derive $k=1.095$, leading to

$\sigma_{z}^{\prime}=112.0 \pm 3.1 \mathrm{~km} \mathrm{~s}^{-1}$.

Comparison of $\sigma_{l}$ and $\sigma_{z}^{\prime}$ leads to $R_{0}=8.07 \pm 0.32 \mathrm{kpc}$.

The error quoted above is the statistical uncertainty. Additionally, systematic errors are introduced by selecting specific rotation profiles, (an)isotropic dispersion profiles, stellar density distributions, and other kinematic parametrizations (see Sect. 5.3). From testing a variety of model parametrizations, we find a systematic error of $0.13 \mathrm{kpc}$. ter of

All in all, we derive a statistical parallax for the nuclear clus-

$R_{0}=8.07 \pm 0.32_{\text {stat }} \pm 0.13_{\text {sys }} \mathrm{kpc}$.

This result is in full agreement with those obtained from the observations of Keplerian stellar orbits around $\mathrm{Sgr} \mathrm{A}^{*}$ (e.g. Eisenhauer et al. 2005; Lu et al. 2006). The most recent values are given by Gillessen et al. (2008) who find $R_{0}=8.14 \pm$ $0.15_{\text {stat }} \pm 0.32_{\text {sys }} \mathrm{kpc}$ and by Ghez et al. (2008) who find $R_{0}=$ $8.0 \pm 0.6_{\text {stat }+ \text { sys }} \mathrm{kpc}$.

Our result also agrees with distance values obtained by earlier statistical parallax measurements, which were $7.9 \pm$ $0.9_{\text {stat }} \mathrm{kpc}$ (Genzel et al. 2000) and, more recently, $7.1 \pm$ $0.7_{\text {stat }} \mathrm{kpc}$ (Eisenhauer et al. 2003a). In the aforementioned experiments the systematic difference between the dispersions in $l$ and $b$ directions was masked by larger statistical uncertainties; therefore, the influence of cluster rotation was not recognized.

Other experiments are based on precision stellar photometry (e.g. Paczynski \& Stanek 1998; McNamara et al. 2000). Recent results have been $R_{0}=7.52 \pm 0.10_{\text {stat }} \pm 0.35_{\text {sys }} \mathrm{kpc}$ by Nishiyama et al. (2006) and $R_{0}=7.94 \pm 0.37_{\text {stat }} \pm 0.26_{\text {sys }} \mathrm{kpc}$ by Groenewegen et al. (2008). All these results are in full agreement with our value.

Another method used to derive $R_{0}$ is the spatial distribution of globular clusters in the Milky Way. In a recent analysis, Bica et al. (2006) examined a sample of 116 clusters and derived $R_{0}=7.2 \pm 0.3_{\text {stat }} \mathrm{kpc}$. Within errors, this is only marginally in agreement with our findings; but when taking into account that Bica et al. (2006) do not provide an estimate for the systematic uncertainty of their method, this deviation is not significant either.

In total, we can conclude the following: (1) the statistical parallax is a valuable independent method for deriving $R_{0}$. (2) Our value and recent measurements based on different approaches (stellar orbits, stellar photometry, distribution of globular clusters) are in good agreement.

\subsection{Acceleration upper limits}

The strong influence of Sgr A* allows the description of the innermost part (few arcsec) of the cluster as a system of massless test particles moving around a point mass on Keplerian orbits. Indeed, orbits located in the innermost $\approx 0.5^{\prime \prime}-$ in the so-called "S-star" group - have been observed now for several years without detecting any significant deviation from a point mass potential (Eisenhauer et al. 2005; Lu et al. 2006; Gillessen et al. 2008; Ghez et al. 2008). When using the very accurate proper motions obtained from the small scale images (typical uncertainties $\approx 4 \mathrm{~km} \mathrm{~s}^{-1}$, see Fig. 4 ), it is possible to detect (or exclude) accelerations in stellar motion as far out as several arcseconds in projected distance.

In this section and Sect. 5.7 we analyse all available stars regardless of their spectral type. We can do this because we 
treat stars individually. Global statistical properties like isotropy, which are affected by the non-relaxed early-type population, do not influence this analysis.

In order to obtain acceleration limits we analysed the proper motions of stars in the small scale fields within $\pm 7^{\prime \prime}$ in RA and Dec from Sgr A*. All star positions were transformed into coordinates radial $\left(q_{\|}\right)$and tangential $\left(q_{\perp}\right)$ to their average position vector. In analogy to the determination of proper motions we fit the star positions $\mathbf{q}=\left(q_{\|}, q_{\perp}\right)$ vs. time $t$ as parabolas of the form

$\mathbf{q}(t)=\mathbf{u} t^{2}+\mathbf{v} t+\mathbf{w}$.

Obviously, this approach delivers the (constant) stellar accelerations $\mathbf{a}(\mathrm{via} \mathbf{a}=2 \mathbf{u}) ; \mathbf{v}$ and $\mathbf{w}$ correspond to velocities and positions at $t=0$ respectively.

Whether a given star shows a significant acceleration depends on the goodness-of-fit of the two physically realistic models, which are (1) a linear proper motion, and (2) an accelerated parabolic motion pointing towards $\operatorname{Sgr} A^{*}$ (i.e. a significantly non-zero value for $\left.a_{\|}\right)$. For both models the respective reduced $\chi^{2}$ (hereafter $\chi_{\text {lin }}^{2}$ for the linear, $\chi_{\text {acc }}^{2}$ for the accelerated case) is computed.

In order to decide if the difference between the two models is significant we make use of the fact that the quantity

$f=\frac{\chi_{\text {lin }}^{2}}{\chi_{\text {acc }}^{2}}$

follows an F-distribution and can thus be examined using an F-test (e.g. Müller 1975; Lehn \& Wegmann 1982). For a given significance level $S \in[0,1]$ and the case of a one-tailed test (e.g. Snedecor \& Cochran 1989) the difference is considered to be significant if $f>F_{m, n, 1-s}$; here $m, n$ are the respective degrees of freedom, $s=1-S$ is the false alarm probability. For the typical case $m=n+1=35$ we find $F_{35,34,0.99}=2.25$ for $S=0.99$. This means that a $99 \%$ confidence detection of non-linear motion requires $f>2.25$.

Our analysis included a total of 755 stars. For five of them significant (using a 99\% confidence limit) accelerations were detected. Three stars were $S$-stars with known orbits. Two more were false positives whose centroids were systematically displaced towards bright neighbouring sources. Due to the high crowding in the innermost arcseconds of the field most of the $S$-stars known to follow Keplerian orbits had to be excluded from this automated analysis and therefore do not contribute.

For the remaining 750 sources, acceleration upper limits are given. Upper limits with a $99 \%$ confidence $(2.58 \sigma)$ are defined as

$\bar{a}_{\|}=\left|a_{\|}\right|+2.58 \times \delta a_{\|}$.

Here $\left|a_{\|}\right|$is the amount of radial acceleration computed according to Eq. (34) and $\delta a_{\|}$its $1 \sigma$ error $^{8}$. Figure 19 shows the resulting limits vs. projected distances. For comparison, we show the acceleration a star would experience if its projected distance were equal to its physical distance. A few limits (5 out of 750) fall below the theoretical line; in these cases, the physical distances need to be larger than the projected distances.

An acceleration upper limit constrains the minimum physical distance $R_{\min }$ of a star. We can compare this number with the

\footnotetext{
8 Even if a star does not show significant acceleration, a parabolic fit finds a (usually non-zero) result for the 2 nd-order term and a corresponding fit error. These values we use here.
}

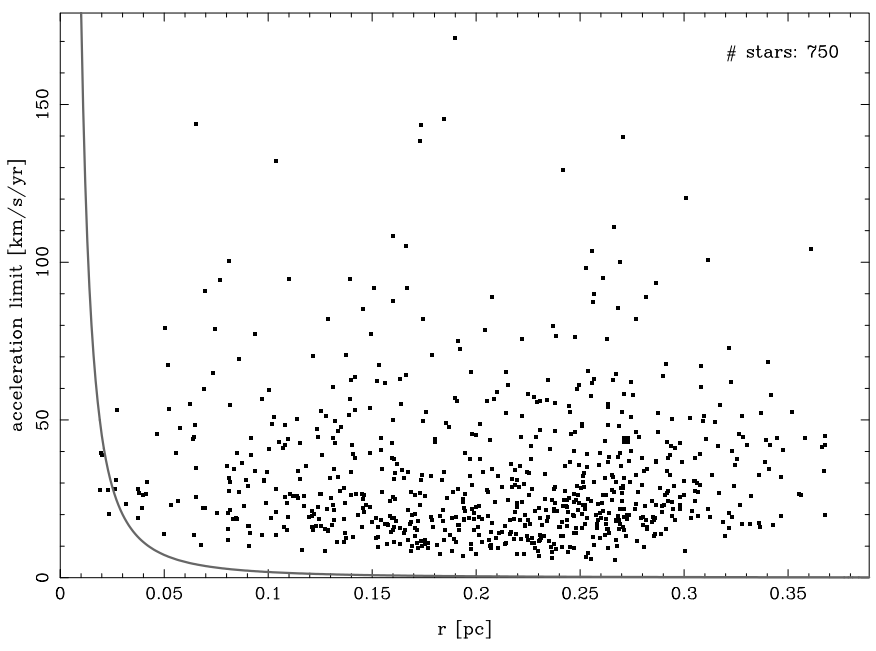

Fig. 19. Acceleration upper limits (99\% confidence level) vs. projected distance $r$ from Sgr A*. Dots mark measured values. The continuous line corresponds to the acceleration a star would experience if its physical distance from Sgr A* would equal its projected distance. The distance scale is $1 \mathrm{pc} \equiv 25.8^{\prime \prime}$. This sample includes 750 of 755 analysed stars. Stars below the line have a physical distance that is necessarily larger than the projected distance.

Table 2. Minimum distance from the plane of Sgr A*, $|z|_{\min }$, vs. projected radius $r$ for five stars with $R_{\min }>r$. The values are based on $2.58 \sigma$ (99\% confidence level) acceleration upper limits. Only stars with $r<0.024$ pc are sufficiently constrained; see Fig. 19.

\begin{tabular}{lcc}
\hline \hline Star ID & $r[\mathrm{mpc}]$ & $|z|_{\min }[\mathrm{mpc}]$ \\
\hline 823 & 19.2 & 12.9 \\
832 & 23.2 & 8.6 \\
1333 & 20.1 & 6.4 \\
1760 & 19.4 & 7.3 \\
3439 & 23.5 & 14.5 \\
\hline
\end{tabular}

star's sky-projected 2D distance $r$. Starting from the amount of sky-projected acceleration

$a_{2 \mathrm{D}}=\frac{G M}{R^{3}} r$

and replacing $a_{2 \mathrm{D}}$ by $\bar{a}_{\|}$we obtain

$R>\left(\frac{G M}{\bar{a}_{\|}} r\right)^{1 / 3} \equiv R_{\min }$

where $R$ is the physical 3D distance from $\operatorname{Sgr} \mathrm{A}^{*}$. If $R_{\min }>r$ for a star, we can derive its minimum distance from the plane of Sgr A* along the line of sight

$|z|_{\min }=\sqrt{R_{\min }^{2}-r^{2}}$

In our case, we have five stars with $R_{\min }>r$ for which we can derive the corresponding $|z|_{\text {min }}$; all have $r<0.024 \mathrm{pc}$. The resulting distribution is presented in Table 2; the largest distance we find is $|z|_{\min } \approx 0.015 \mathrm{pc}$.

Given the good accuracies of the stellar proper motions (few $\mathrm{km} \mathrm{s}^{-1}$ ), the large values for the acceleration limits (tens of $\mathrm{km} \mathrm{s}^{-1} / \mathrm{yr}$; see Fig. 19) might not be obvious. However, this is 
given by the fact that both velocities and accelerations are derived from position measurements. Whereas the statistical error of a velocity measurement scales like

$\delta v \propto \delta q \times t^{-1}$,

the statistical error of an acceleration measurement scales like

$\delta a \propto \delta q \times t^{-2}$

with $\delta q$ being the typical position measurement uncertainty, $t$ being the total time covered by data. From this, we see that on the one hand a timeline of five years is sufficient for precision proper motion measurements. On the other hand, the same timeline of five years is not yet sufficient to constrain the $z$ coordinate of more than a handful of stars. However, as $\delta a \propto t^{-2}$, already a moderate extension of the timeline leads to a substantial improvement.

From this we can draw important conclusions affecting future analyses and observing strategies. (1) Future works should include earlier data. For the GC, we have data at hand starting as early as 1992, taken with the SHARP I speckle imaging camera at the ESO-NTT (e.g. Genzel et al. 1996). For the case of the $S$-stars orbiting Sgr A*, this dataset has been used with great success (Schödel et al. 2003; Gillessen et al. 2008). (2) Continued NACO observation of the nuclear cluster will provide valuable new insights. Therefore the monitoring of the cluster should be continued.

\subsection{The star group IRS13E}

An object of special interest is the star group IRS13E, located $3^{\prime \prime}$ west and 1.5" south of Sgr A*. This object consists of three bright $(H \approx 13)$ main components concentrated within a region of about $0.2^{\prime \prime}$ radius. They surround fainter objects which are probably blends of several point sources. Paumard et al. (2006) found a significant stellar density excess in the immediate vicinity $\left(0.7^{\prime \prime}\right)$ of the three main stars and identified the IRS13E group as a star cluster. Based on stability arguments with respect to the tidal field of Sgr A*, the possibility that IRS13E hosts an intermediate-mass black hole was previously discussed by Maillard et al. (2004).

In order to examine this scenario in more detail, we tested whether stars within a radius of $0.7^{\prime \prime}$ are kinematically connected with the three central sources. We extracted proper motions for the three main components and an additional 17 stars with magnitudes down to $H \approx 19.5$ (for a similar analysis using the proper motions of another set of stars, see Schödel et al. 2005). In the following, we call the three main stars "set A" and the 17 field stars "set B". As the target area is too crowded to be fully covered by the automatic procedures described in Sect. 3, we extracted image positions manually. We applied the PSF fitting routine StarFinder by Diolaiti et al. (2000) and checked the interepoch source identifications by eye. Our analysis included seven very good $H$ and $K$-band small scale images obtained between 2002 and 2007.

The main results are summarized in Fig. 20. The top panel shows all stellar proper motions with respect to the standard astrometric reference frame tied to the GC cluster, i.e. co-moving with the GC cluster (see Sect. 3). The bottom panel of this figure shows the same proper motions in a reference frame comoving with set $\mathrm{A}$, using the average motion of set $\mathrm{A}$ as the zero-point. In the latter case, almost all stars show a motion directed from west to east, as if IRS13E were moving through a separate, non-co-moving foreground/background population. In
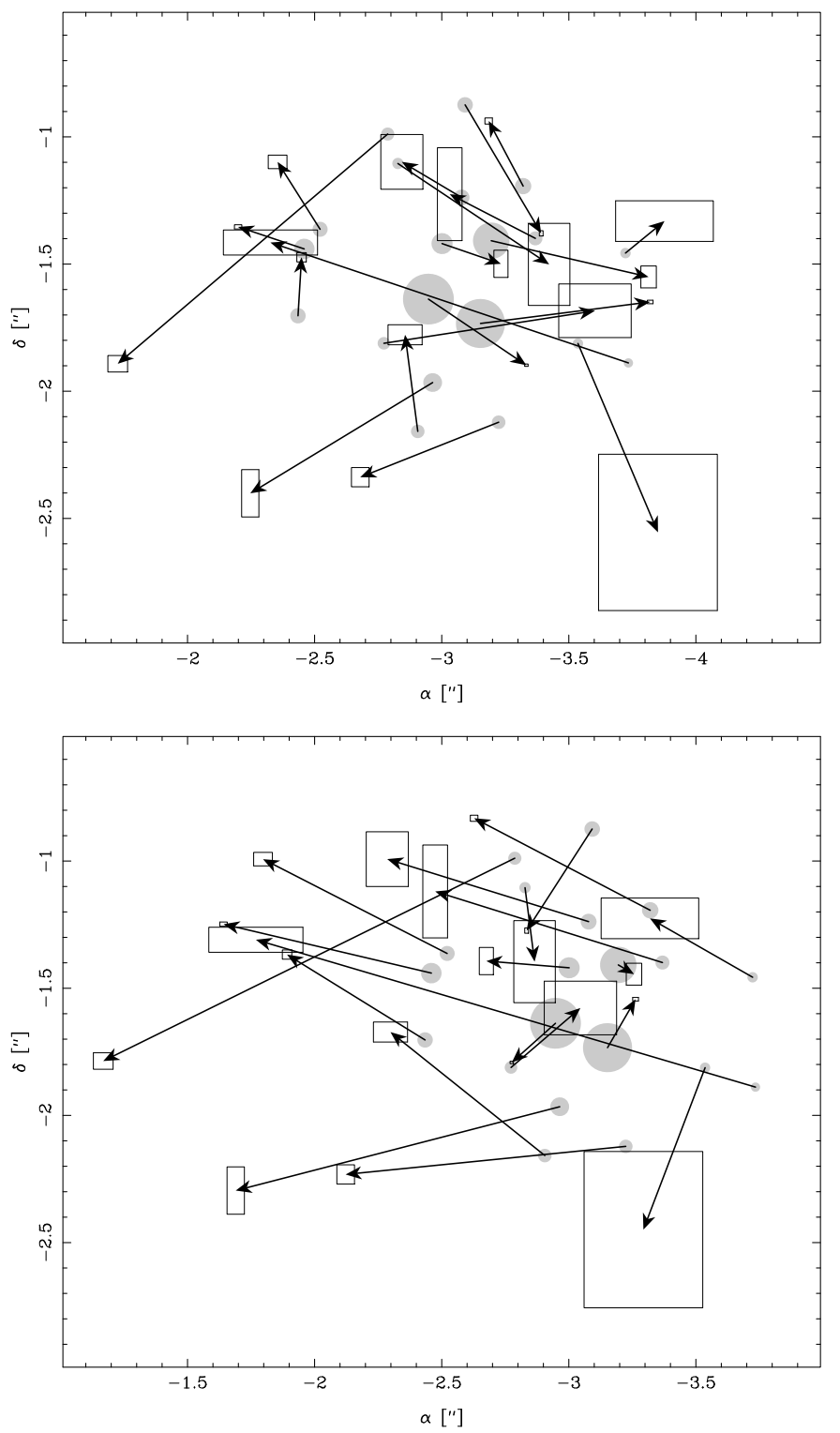

Fig. 20. Proper motion maps for the IRS13E group. Filled circles are stars, circle diameters are proportional to the 0.25 th power of $H$-band fluxes. Coordinates are $\alpha, \delta$ in arcsec relative to Sgr A*; please note the shift in $\alpha$. Arrows are proper motions, 1 unit in length corresponds to $10 \mathrm{mas} / \mathrm{yr}$. Boxes around arrow heads indicate the $1-\sigma$ velocity uncertainties. Top panel: proper motions co-moving with the GC cluster as defined in Sect. 3. Bottom panel: proper motions co-moving with the central three brightest stars. A streaming motion of the three brightest stars relative to a foreground/background population can be seen. Apparently, only a small fraction, if any, of the examined stars are bound to the IRS13E group.

$\alpha$ (or $x$ ) direction, the average motion (co-moving with set A) of set $\mathrm{B}$ is $\left\langle v_{x}\right\rangle=7.1 \pm 1.3 \mathrm{mas} / \mathrm{yr}$, wheras the velocity dispersion is $\sigma_{x}=5.3 \pm 0.9 \mathrm{mas} / \mathrm{yr}$. In $\delta$ (or $y$ ), we find $\left\langle v_{y}\right\rangle=0.6 \pm$ $0.9 \mathrm{mas} / \mathrm{yr}, \sigma_{y}=3.7 \pm 0.7 \mathrm{mas} / \mathrm{yr}$. These numbers show a clear streaming of the three main stars with respect to the 17 field stars.

For their analysis, Paumard et al. (2006, see especially their Fig. 9) used stellar number counts in the IRS13E region down to magnitudes $H=20.4$. They compared the surface densities inside $\left(\Sigma_{<}\right)$and outside $\left(\Sigma_{>}\right)$a projected radius of $p=0.68^{\prime \prime}$ 
from the centre of set A. Counting all $H<20.4$ stars they found

$\Sigma_{<}=31.7 \pm 4.7 \operatorname{arcsec}^{-2}$

$\Sigma_{>}=13.1 \pm 1.2 \operatorname{arcsec}^{-2}$.

This corresponds to a $4.3 \sigma$ excess of stars within $p$. Using a more conservative limit of $H<19.4$, the resulting densities are

$\Sigma_{<}=17.9 \pm 3.5 \operatorname{arcsec}^{-2}$

$\Sigma_{>}=7.9 \pm 0.9 \operatorname{arcsec}^{-2}$

corresponding to a $2.8 \sigma$ excess of stars within $p$. All errors given for numbers and densities are Poisson errors. The total number of $H<19.4$ stars located within $p$ is 26 . This magnitude cut corresponds (within the errors) to the magnitude limit of our set $B$.

We used the kinematic information obtained for the set B stars to follow up on the surface density analysis by Paumard et al. (2006). We recalculated the surface density of $H<$ 19.4 stars, but excluded stars which are too fast with respect to set A. The star selection was done in two steps. First, we computed the $2 \mathrm{D}$ velocity dispersion of the three set A stars. This dispersion was $\sigma_{\mathrm{A}}=1.9 \pm 0.8 \mathrm{mas} / \mathrm{yr}$.

Then we computed the bias-corrected 2D speeds $v_{2 \mathrm{D}}$ of the set $\mathrm{B}$ stars relative to set A (reference frame co-moving with set A). We identified all set B stars (a) located within $p$ and (b) showing $v_{2 \mathrm{D}}>3 \sigma_{\mathrm{A}}$. For these stars we assumed that they cannot be physically connected to the set A stars.

We found nine stars satisfying criteria (a) and (b). Excluding them from the sample of Paumard et al. (2006) reduces the number of $H<19.4$ stars located within $p$ from 26 to 17 . Recalculating the surface density leads to

$\Sigma_{<}=11.7 \pm 2.8 \operatorname{arcsec}^{-2}$.

The difference of densities inside and outside $p$ then becomes

$\Delta \Sigma=\Sigma_{<}-\Sigma_{>}=3.8 \pm 3.0 \operatorname{arcsec}^{-2}$

corresponding to a significance of $1.3 \sigma$ for a deviation from zero. We therefore do not see a significant excess of $H<19.4$ stars within $p$.

We can conclude that our kinematic analysis seriously weakens the scenario proposing that IRS13E is the core of a substantial star cluster.

\section{Conclusions}

In this article we analysed and discussed the kinematic properties of the Galactic centre $\mathrm{CO}$ absorption line star cluster. This work is based on adaptive optics assisted diffraction-limited near-infrared imaging and integral-field spectroscopy. We collected proper motions for 5445 stars, 3D velocities for 664 stars, and acceleration limits for 750 stars. Our analysis led to the following main results:

1. The cluster shows a global rotation in the sense of general Galactic rotation.

2. The stellar 3D speed distributions can be locally approximated by Maxwellians. This confirms the relaxed nature of the CO star cluster.

3. We find one high-velocity star with a $3 \mathrm{D}$ speed $v_{3 \mathrm{D}}=810 \pm$ $9 \mathrm{~km} \mathrm{~s}^{-1}$ which might be unbound. It might have been ejected from the cluster by three-body interactions with SgrA*.
4. We obtain a deprojected 3D description of the cluster kinematics. We fit the observed velocity dispersion profiles $\sigma_{l, b}(r)$ and the rotation curve $\left\langle v_{z}\right\rangle(l)$. The data are described well by assuming a $\rho(R) \propto R^{-2}$ sphere density profile and global rotation. From the model solution, we extract the cluster's mass profile out to $M_{*} \simeq 2.3 \times 10^{7} M_{\odot}$ at $R \simeq 4$ pc.

5. The two-point correlation function of the stellar 4D phasespace positions agrees with that of a uniform isotropic rotator within $4 \%$. We find no obvious indication for phasespace subtructure like star streams. From this we conclude qualitatively that there has been no major distortion of the GC cluster within the last few $10^{5}$ years.

6. Using the $3 \mathrm{D}$ velocity dispersion, we derive an improved statistical parallax to the GC of $R_{0}=8.07 \pm 0.32_{\text {stat }} \pm$ $0.13_{\text {sys }} \mathrm{kpc}$. This result is in good agreement with the values obtained by stellar orbit, stellar photometry, and globular cluster distribution studies.

7. For stars located within the innermost few (projected) arcsec, we calculate limits on accelerations in the plane of sky. We use these limits to constrain the stars' minimum line-of-sight distances from the plane of $\mathrm{Sgr} \mathrm{A}^{*}$. We find non-trivial results for 5 out of 750 stars and conclude that already a moderate extension of the observation timeline can increase this number substantially.

8. The star group IRS13E does not co-move with the $H<$ 19.4 stars in its $0.7^{\prime \prime}$ vicinity. When excluding stars which are too fast to be part of the IRS13E system, there is no sign for a significant star concentration. This seriously weakens the case for IRS13E being the core of a substantial star cluster.

In summary, our analysis has improved substantially our knowledge regarding the kinematic properties of the GC star cluster. The next step will be to feed our extensive data set into a fullscale dynamical model. We plan to make use of the recently developed NMAGIC code (De Lorenzi et al. 2007) in order to finalize the physical description of the old stellar population in the central parsec of our Milky Way.

Acknowledgements. Special thanks to Nancy Ageorges (ESO, MPE) for helpful discussions of the instrumental geometric distortion and registration of NACO images. We are grateful to Tal Alexander and Hagai Perets (both: Weizmann Institute of Science, Rehovot, Israel) for enlightening discussions of stellar dynamics. Many thanks to Nicolas Bouché (MPE) for his description of the properties of two-point correlation functions, and to Rainer Schödel (IAA Grenada) for helpful discussions of the GC cluster mass profile. We are grateful to Hauke Engel, Eva Noyola, and Richard Davies (all: MPE) for helpful discussions of the properties of the Jeans equation. S.T. would like to thank Markus Schmaus (LMU Munich, Dept. of Mathematics) for pointing out the concept of stochastic optimization. And last but not least we are grateful to the referee, James Binney, whose comments helped to improve the quality of this article.

\section{Appendix A: Image distortion parameters}

We extracted the distortion parameters from our imaging data by executing the following steps:

1. Combination of all individual frames to be mosaicked via simple shift-and-add (SSA) with integer-pixel accuracy.

2. Constructing a list of many $(\approx 200)$ good (meaning bright, but unsaturated stars well separated from neighbouring sources) reference stars distributed over the entire FOV. For source selection, the SSA image is used.

3. Re-identification of all reference stars located in the FOV of each individual image, followed by determining their detector positions.

4. Computation of all pairwise star-star separations in each image. This calculation results in a net of baselines for each 
image. Baselines present in more than one image are subject to inter-image comparison.

5. Modelling the distortion correction.

The three model parameters $\left(\beta, x_{C}, y_{C}\right)$ were fit by a $\chi^{2}$ minimization. Geometric distortion implies that the detector plate scale is a function of the detector position; thus the length of a given baseline (in units of pixels) depends on its location on the detector. The optimum parameter set is found by iteratively comparing all baselines in all images shifted on sky, applying the temporary parameters to the reference star detector coordinates, and checking for the improvement.

In case of the large scale (27 mas/pixel) images we executed parameter fits in a straight forward manner. We made use of the analytic fit engine FindMinimum implemented in the software package Mathematica.

For the small scale (13 mas/pixel) data sets this procedure was not applicable. Due to the less significant distortion and the smaller number of reference stars available $(\approx 100)$, the analytic fit algorithm usually did not converge towards a reliable result. Thus we constructed a stochastic minimization algorithm which searches the parameter space iteratively using the following scheme:

1. Compute the value of the cost function (i.e. the function to be minimized) at the actual position in parameter space.

2. Select a second position in parameter space and compute the value of the cost function at that position.

3. If the value of the cost function at the new position is smaller than the actual one: move there. Otherwise: stay at present position.

4. Repeat steps 1-3 until a fixed number of iterations is completed.

Starting from a given initial position in parameter space, in iteration $n$ for each parameter $p$ a new value (step 2) is computed as

$p_{n+1}=p_{n}+s(n) \cdot\left(1 / z_{[0,1]}-1\right) \cdot \epsilon\left(0.5-z_{[0,1]}\right)$

with

$s(n)=s_{0} \cdot 10^{-n / N}$.

Here $N$ is the maximum number of iterations, $z_{[0,1]}$ a random number in the range $[0,1], \epsilon(x)$ the sign function returning -1 or +1 depending on the sign of $x$, and $s_{0}$ the initial step size.

This definition assures that (1) the algorithm cannot be easily trapped in a local minimum, as even extreme search radii are occasionally tested, and (2) the vicinity of the best-so-far-solution found at the end of the search time is explored with reasonable accuracy, as the average search radius decreases exponentially with time (thus increasing the "selection pressure" on the algorithm). The idea for this definition was taken from the concept of Simulated Annealing introduced by Kirkpatrick et al. (1983).

\section{Appendix B: Accuracy of radio coordinates}

Absolute radio coordinates and infrared image coordinates are tied via nine $\mathrm{SiO}$ maser stars in the field of view. These maser stars show finite statistical errors in both radio and NIR image positions. The uncertainties limit the accuracy of the global coordinates.

We examined the influence of these uncertainties on the transformation accuracy using a Monte-Carlo test. We executed $10^{5}$ coordinate transformations, each time using sets of positions with random displacements. The displacements followed
Gaussian distributions according to the individual statistical errors.

By sampling a coordinate grid with the typical FOV size (positions $\pm 20^{\prime \prime}$ from Sgr A*) we mapped the transformation uncertainty as a function of position. The results are shown in Fig. 3. The contours mirror the geometry of the alignment of the reference stars in the plane of the sky (see also Fig. 1); the errors vary in the range $1.2 \ldots 5$ mas.

Of particular interest is the accuracy of the global position $(0,0)$ which corresponds to the location of Sgr A*, the dynamical centre of the GC star cluster. Here the errors were

$\delta \mathrm{RA}=1.26$ mas

$\delta \mathrm{Dec}=1.20$ mas.

These errors are uncertainties in absolute positions. Our kinematic analysis is based on time-resolved relative positions. Therefore the proper motion accuracies are much better than suggested by the errors of the absolute positions.

\section{References}

Alexander, T. 2005, PhR, 419, 65

Anderson, J., Bedin, L. R., Piotto, G., Yadav, R. S., \& Bellini, A. 2006, A\&A, 454, 1029

Balick, B., \& Brown, R. L. 1974, ApJ, 194, 265

Bender, R., Kormendy, J., Bower, G., et al. 2005, ApJ, 631, 280

Bica, E., Bonatto, C., Barbuy, B., \& Ortolani, S. 2006, A\&A, 450, 105

Bindoni, D., \& Secco, L. 2008, NewAR, 52, 1

Binney, J., \& Tremaine, S. 1987, Galactic Dynamics (Princeton University Press) Bonanos, A. Z., López-Morales, M., Hunter, I., \& Ryans, R. S. I. 2008, ApJ, 675, L77

Bonnet, H., Ströbele, S., Biancat-Marchet, F., et al. 2003, SPIE, 4839, 329

Bonnet, H., Abuter, R., Baker, A., et al. 2004, The ESO Messenger, 117, 17

Brown, W. R., Geller, M. J., Kenyon, S. J., \& Kurtz, M. J. 2005, ApJ, 622, L33

Brown, W. R., Geller, M. J., Kenyon, S. J., Kurtz, M. J., \& Bromley, B. C. 2007 , ApJ, 671, 1708

Brown, W. R., Geller, M. J., \& Kenyon, S. J. 2008, ApJ, submitted

Cox, A. N. 2000, Allen's Astrophysical Quantities, 4th edn. (New York: Springer)

Davis, M., \& Peebles, P. J. E. 1983, ApJ, 267, 465

De Lorenzi, F., Debattista, V. P., Gerhard, O., \& Sambhus, N. 2007, MNRAS, 376, 71

Diolaiti, E., Bendinelli, O., Bonaccini, D., et al. 2000, A\&AS, 147, 335

Eckart, A., \& Genzel, R. 1997, MNRAS, 284, 576

Edelmann, H., Napiwotzki, R., Heber, U., Christlieb, N., \& Reimers, D. 2005, ApJ, 634, L181

Eisenhauer, F., Schödel, R., Genzel, R., et al. 2003a, ApJ, 597, L121

Eisenhauer, F., Abuter, R., Bickert, K., et al. 2003b, SPIE, 4841, 1548

Eisenhauer, F., Tecza, M., Thatte, N., et al. 2003c, The ESO Messenger, 113, 17

Eisenhauer, F., Genzel, R., Alexander, T., et al. 2005, ApJ, 628, 246

Ferrarese, L., \& Ford, H. 2005, SSRv, 116, 523

Figer, D. F., Gilmore, D., Kim, S. S., et al. 2003, ApJ, 599, 1139

Genzel, R., Thatte, N., Krabbe, A., Kroker, H., \& Tacconi-Garman, L. E. 1996, ApJ, 472, 153

Genzel, R., Eckart, A., Ott, T., \& Eisenhauer, F. 1997, MNRAS, 291, 219

Genzel, R., Pichon, C., Eckart, A., Gerhard, O. E., \& Ott, T. 2000, MNRAS, 317, 348

Genzel, R., Schödel, R., Ott, T., et al. 2003, ApJ, 594, 812

Gillessen, S., et al. 2008, ApJ, submitted

Gerhard, O. E. 1994, in Lecture Notes in Physics, ed. G. Contopoulos, \& N. K. Spyrou (Springer Verlag)

Gerhard, O. 2001, ApJ, 546, L39

Ghez, A. M., Klein, B. L., Morris, M., \& Becklin, E. E. 1998, ApJ, 509, 678

Ghez, A. M., Duchêne, G., Matthews, K., et al. 2003, ApJ, 586, L127

Ghez, A. M., Salim, S., Hornstein, S. D., et al. 2005, ApJ, 620, 774

Ghez, A. M., et al. 2008, ApJ, in press

Goodman, J. 2003, MNRAS, 339, 937

Groenewegen, M. A. T., Udalski, A., \& Bono, G. 2008, A\&A, 481, 441

Gustafsson, B., Edvardsson, B., Eriksson, K., et al. 2003, ASP Conf. Ser., 288, 331 
Hargreaves, J. C., Gilmore, G., Irwin, M. J., \& Carter, D. 1994, MNRAS, 269, 957

Hartung, M., Lenzen, R., Hofmann, R., et al. 2003, SPIE, 4841, 425

Hills, J. G. 1988, Nature, 331, 687

Hirsch, H. A., Heber, U., O’Toole, S. J., \& Bresolin, F. 2005, A\&A, 444, L61

Ibata, R., Irwin, M., Lewis, G. F., \& Stolte, A. 2001, ApJ, 547, L133

Jähne, B. 2005, Digital Image Processing (Heidelberg: Springer Verlag)

Kerr, F. J., \& Lynden-Bell, D. 1986, MNRAS, 221, 1023

Kirkpatrick, S., Gelatt, C. D., \& Vecchi, M. P. 1983, Science, 220, 4598

Kormendy, J., \& Richstone, D. 1995, ARA\&A, 33, 581

Kormendy, J., \& Bender, R. 1999, ApJ, 522, 772

Krabbe, A., Genzel, R., Eckart, A., et al. 1995, ApJ, 447, L95

Launhardt, R., Zylka, R., \& Mezger, P. G. 2002, A\&A, 384, 112

Lehn, J., \& Wegmann, H. 1982, Einführung in die Statistik (Darmstadt: Wissenschaftliche Buchgesellschaft)

Levin, Y., \& Beloborodov, A. M. 2003, ApJ, 590, L33

Lindqvist, M., Habing, H. J., \& Winnberg, A. 1992, A\&A, 259, 118

Lu, J. R., Ghez, A. M., Hornstein, S. D., et al. 2006, JPhCS, 54, 279

Lynden-Bell, D. 1967, MNRAS, 136, 101

Lynden-Bell, D., \& Rees, M. J. 1971, MNRAS, 152, 461

Maillard, J. P., Paumard, T., Stolovy, S. R., \& Rigaut, F. 2004, A\&A, 423, 155

Maness, H., Martins, F., Trippe, S., et al. 2007, ApJ, 669, 1024

Martinez-Delgado, D., Gómez-Flechoso, M. Á., Aparicio, A., \& Carrera, R.2004, ApJ, 601, 242

McGinn, M. T., Sellgren, K., Becklin, E. E., \& Hall, D. N. B. 1989, ApJ, 338, 824

McMillan, S. L. W., \& Portegies Zwart, S. F. 2003, ApJ, 596, 314
McNamara, D. H., Madsen, J. B., Barnes, J., \& Ericksen, B. F. 2000, PASP, 112 , 202

Montenbruck, O., \& Pfleger, T. 1989, Astronomie mit dem Personal Computer (Heidelberg: Springer Verlag)

Mouawad, N., Eckart, A., Pfalzner, S., et al. 2005, AN, 326, 83

Müller, P. H. 1975, Lexikon der Wahrscheinlichkeitsrechnung und Mathematischen Statistik (Berlin: Akademie-Verlag)

Nishiyama, S., Nagata, T., Sato, S., et al. 2006, ApJ, 647, 1093

Paczynski, B., \& Stanek, C. 1998, ApJ, 494, L129

Paumard, T., Genzel, R., Martins, F., et al. 2006, ApJ, 643, 1011

Perets, H. B., Hopman, C., \& Alexander, T. 2007, ApJ, 656, 709

Przybilla, N., Nieva, M. F., Heber, U., et al. 2008, A\&A, 480, L37

Reid, M., Menten, K. M., Trippe, S., Ott, T., \& Genzel, R. 2007, ApJ, 659, 378

Rousset, G., Lacombe, F., Puget, P., et al. 2003, SPIE, 4839, 140

Schödel, R., Ott, T., Genzel, R., et al. 2002, Nature, 419, 694

Schödel, R., Ott, T., Genzel, R., et al. 2003, ApJ, 596, 1015

Schödel, R., Eckart, A., \& Iserlohe, C. 2005, ApJ, 625, L111

Schödel, R., Eckart, A., Alexander, T., et al. 2007, A\&A, 469, 125

Seabroke, G. M., \& Gilmore, G. 2007, MNRAS, 380, 1348

Snedecor, G. W., \& Cochran, W. G. 1989, Statistical Methods, 8th edn. (Iowa State University Press)

Stetson, P. B. 1987, PASP, 99, 191

Tonry, J., \& Davis, M. 1979, AJ, 84, 1511

Wilson, C. P. 1975, AJ, 80, 175

Yanny, B., Newberg, H. J., Grebel, E. K., et al. 2003, ApJ, 588, 824

Zhu, Q., et al. 2008, ApJ, in press 